\title{
On the Range of Validity of the Dipole Picture
}

\author{
Carlo Ewerz,, , * Andreas von Manteuffel,, ,, and Otto Nachtmann ${ }^{2}$, \\ ${ }^{1} E C T^{*}$, Strada delle Tabarelle 286, I-38050 Villazzano (Trento), Italy \\ ${ }^{2}$ Institut für Theoretische Physik, Universität Heidelberg, \\ Philosophenweg 16, D-69120 Heidelberg, Germany
}

\author{
Ein Photon kommt recht schnell daher \\ und glaubt, daß es ein Dipol wär. \\ Ein Proton aus recht großer Ferne \\ sieht dies nur allzu gerne. \\ Und was die zwei dann treiben, \\ kann keine Theorie beschreiben, \\ Gluonen fliegen hin und her, \\ das versteht kein Mensch nicht mehr. \\ Viel komplizierter als gedacht \\ ist doch die QCD gemacht. \\ Schon die Sache mit dem Dipol war - \\ so ist es wohl - nicht ganz so klar. \\ Der Phänomenologe, keine Frage, \\ inspiziert trotzdem die Datenlage. \\ Modelle sind dann schnell zur Hand, \\ vielleicht sogar mit Fehlerband. \\ Und kommt die Kurve an die Punkte ran, \\ weht niemanden ein Zweifel an. \\ Auf daß das nicht so bleiben soll, \\ schreibt unsereins dies Paper voll.
}

\begin{abstract}
We derive correlated bounds on ratios of deep inelastic structure functions from the dipole picture of photon-hadron scattering at high energies. In particular we consider ratios of the longitudinal structure function, the total structure function and the charm part of the latter. We also consider ratios of total structure functions taken at the same energy but at three different photon virtualities. It is shown that by confronting these bounds with experimental data we can significantly constrain the range of validity of the dipole picture.
\end{abstract}

\section{INTRODUCTION}

The HERA collider has provided a wealth of data on deep inelastic lepton-proton scattering (DIS), in particular on DIS structure functions [1]-[ 5 . Many of these experimental results are analyzed in terms of the dipole picture for quasi-real and virtual photon-proton scattering, see for instance [6]-[15]. The physical idea underlying the dipole picture is that at high energies the quasireal or virtual photon acts like a quark-antiquark dipole [16, 17, 18]. The $\gamma^{*} p$ collision is viewed as a two step process. First the $\gamma^{*}$ dissociates into a $q \bar{q}$ dipole, the distribution of dipole sizes being described by the photon wave function. Then the dipole scatters on the proton. The latter step is considered to be a purely hadronic re-

*Electronic address: C.Ewerz@thphys.uni-heidelberg.de ${ }^{\dagger}$ Electronic address: A.v.Manteuffel@thphys.uni-heidelberg.de

‡Electronic address: O.Nachtmann@thphys.uni-heidelberg.de action. The roots of this picture can be traced back to [19, 20]. For a review see [21]. In two articles [22, 23] the theoretical foundations of the standard dipole picture were examined and the assumptions were spelled out which are necessary in order to arrive at it. Bounds on ratios of DIS structure functions were derived in 24] which must be respected if the standard dipole picture holds. These bounds turn out to be relevant for the important question of the range of applicability of the dipole picture. It was in fact shown in 24] that the comparison of measured data on structure functions with the bounds from the dipole picture can be used to restrict its range of validity.

In the present article we discuss further bounds for DIS observables which follow from the standard dipole picture. We shall, in particular, give correlated bounds for $F_{L} / F_{2}$ versus $F_{2}^{(c)} / F_{2}$. Here $F_{2}, F_{2}^{(c)}$ and $F_{L}$ are the total $F_{2}$ structure function, its charm part and the longitudinal structure function, respectively. Furthermore, we shall consider the structure function $F_{2}\left(W, Q^{2}\right)$ at the same $\gamma^{*} p$ c.m. energy $W$ but at three different values of 
the photon virtuality $Q^{2}$, and we shall derive correlated bounds on ratios of these three values of $F_{2}$. We show that by comparing the bounds found here with measured data one can further restrict the kinematical range of applicability of the dipole picture.

Our paper is organized as follows. In section [II] we fix our notation and recall the main results from [22, 23] which are relevant here. In section III we derive the correlated bounds on the total, longitudinal and charm structure functions. In section $I V$ we discuss the correlated bounds for structure functions at the same $\gamma^{*} p$ c.m. energy and three different values of $Q^{2}$. Section $[\mathrm{V}$ contains our conclusions. In appendix $\mathrm{A}$ we illustrate how our bounds are affected at large $Q^{2}$ by the choice of flux factor in the definition of the $\gamma^{*} p$ cross sections for finite Bjorken- $x$. In appendix $\mathrm{B}$ we explain some mathematical notions and techniques used in the derivation of our bounds.

\section{THE STANDARD DIPOLE PICTURE}

We consider deep inelastic lepton-proton scattering

$$
l(k)+p(p) \rightarrow l\left(k^{\prime}\right)+X\left(p^{\prime}\right)
$$

where $l=e^{-}, e^{+}$. In standard kinematics (see for instance [25]) we have

$$
\begin{aligned}
s & =(p+k)^{2}, \\
q & =k-k^{\prime}=p^{\prime}-p, \\
Q^{2} & =-q^{2} \\
\nu & =p q / m_{p}, \\
W^{2} & =(p+q)^{2}=2 m_{p} \nu-Q^{2}+m_{p}^{2}, \\
y & =\frac{p q}{p k}=\frac{2 m_{p} \nu}{s-m_{p}^{2}}, \\
x & =\frac{Q^{2}}{2 m_{p} \nu} .
\end{aligned}
$$

We consider moderate $Q^{2}$,

$$
0 \leq Q^{2} \lesssim 10^{3} \mathrm{GeV}^{2}
$$

such that only photon exchange has to be taken into account. That is, we are interested in the reaction

$$
\gamma^{*}(q)+p(p) \rightarrow X\left(p^{\prime}\right)
$$

where the proton is supposed to be unpolarized and a sum over all final states $X$ is performed. The total cross section for (2.4) is encoded in the hadronic tensor

$$
\begin{aligned}
& W^{\mu \nu}(p, q)=-W_{1}\left(\nu, Q^{2}\right)\left(g^{\mu \nu}-\frac{q^{\mu} q^{\nu}}{q^{2}}\right) \\
& +\frac{1}{m_{p}^{2}} W_{2}\left(\nu, Q^{2}\right)\left(p^{\mu}-\frac{(p q) q^{\mu}}{q^{2}}\right)\left(p^{\nu}-\frac{(p q) q^{\nu}}{q^{2}}\right)
\end{aligned}
$$

with the usual invariant functions $W_{1,2}$.

In order to define the cross sections for longitudinally and transversely polarized virtual photons in (2.4) we work in the proton rest system, supposing

$$
\left(q^{\mu}\right)=\left(\begin{array}{c}
q^{0} \\
0 \\
0 \\
|\boldsymbol{q}|
\end{array}\right)
$$

and define the following photon polarization vectors:

$$
\begin{aligned}
& \left(\varepsilon_{ \pm}^{\nu}\right)=\mp \frac{1}{\sqrt{2}}\left(\begin{array}{c}
0 \\
1 \\
\pm i \\
0
\end{array}\right), \\
& \left(\varepsilon_{L}^{\nu}\right)=\frac{1}{Q}\left(\begin{array}{c}
|\boldsymbol{q}| \\
0 \\
0 \\
q^{0}
\end{array}\right), \\
& \left(\varepsilon_{L}^{\prime \nu}\right)=\left(\varepsilon_{L}^{\nu}\right)-\frac{\left(q^{\nu}\right)}{Q}=\frac{1}{Q}\left(\begin{array}{c}
|\boldsymbol{q}|-q^{0} \\
0 \\
0 \\
q^{0}-|\boldsymbol{q}|
\end{array}\right) .
\end{aligned}
$$

With Hand's convention [26] the $\gamma^{*} p$ cross sections for transverse or longitudinal $\gamma^{*}$ polarization are

$$
\begin{aligned}
\sigma_{T}\left(W, Q^{2}\right) & =\frac{2 \pi m_{p}}{W^{2}-m_{p}^{2}} \varepsilon_{+}^{\mu *} e^{2} W_{\mu \nu} \varepsilon_{+}^{\nu} \\
& =\frac{2 \pi m_{p}}{W^{2}-m_{p}^{2}} \varepsilon_{-}^{\mu *} e^{2} W_{\mu \nu} \varepsilon_{-}^{\nu} \\
& =\frac{2 \pi m_{p}}{W^{2}-m_{p}^{2}} e^{2} W_{1}\left(\nu, Q^{2}\right) \\
\sigma_{L}\left(W, Q^{2}\right)= & \frac{2 \pi m_{p}}{W^{2}-m_{p}^{2}} \varepsilon_{L}^{\mu *} e^{2} W_{\mu \nu} \varepsilon_{L}^{\prime \nu} \\
= & \frac{2 \pi m_{p}}{W^{2}-m_{p}^{2}} \varepsilon_{L}^{\mu *} e^{2} W_{\mu \nu} \varepsilon_{L}^{\nu} \\
& =\frac{2 \pi m_{p}}{W^{2}-m_{p}^{2}}\left[e^{2} W_{2}\left(\nu, Q^{2}\right) \frac{\nu^{2}+Q^{2}}{Q^{2}}\right. \\
& \left.-e^{2} W_{1}\left(\nu, Q^{2}\right)\right] .
\end{aligned}
$$

Note that due to gauge invariance the hadronic tensor $W^{\mu \nu}(2.5)$ satisfies

$$
\begin{aligned}
& q_{\mu} W^{\mu \nu}(p, q)=0, \\
& W^{\mu \nu}(p, q) q_{\nu}=0 .
\end{aligned}
$$

Thus in the definition of $\sigma_{L}$ (2.11) it is irrelevant whether we choose the $\gamma^{*}$ polarization vector as $\varepsilon_{L}^{\nu}$ (2.8) or $\varepsilon_{L}^{\prime \nu}$ (2.9). However, as shown in [23], in applications of the dipole model it is essential to use $\varepsilon_{L}^{\prime \nu}$ and not $\varepsilon_{L}^{\nu}$, in particular when one calculates the photon wave function from the Feynman rule for an incoming photon splitting into outgoing on-shell quark and antiquark. In that case the 
photon polarization vector has to be chosen such that its components remain finite in the high energy limit, as is true for $\varepsilon_{L}^{\prime}$ but not for $\varepsilon_{L}$.

The standard structure function $F_{2}$ is defined as

$$
\begin{aligned}
& F_{2}\left(W, Q^{2}\right)=\nu W_{2}\left(\nu, Q^{2}\right) \\
& =\frac{Q^{2}}{4 \pi^{2} \alpha_{\mathrm{em}}}\left[\sigma_{T}\left(W, Q^{2}\right)+\sigma_{L}\left(W, Q^{2}\right)\right] \\
& \quad \times\left\{1+\frac{Q^{2}\left(W^{2}+Q^{2}+3 m_{p}^{2}\right)}{\left(W^{2}-m_{p}^{2}\right)\left(W^{2}+Q^{2}-m_{p}^{2}\right)}\right\}^{-1} \\
& =\frac{Q^{2}}{4 \pi^{2} \alpha_{\mathrm{em}}}\left[\sigma_{T}\left(W, Q^{2}\right)+\sigma_{L}\left(W, Q^{2}\right)\right](1-x) \\
& \quad+\mathcal{O}\left(m_{p}^{2} / W^{2}\right) .
\end{aligned}
$$

In the high energy limit, $W \gg Q, m_{p}$, this simplifies to the commonly used form

$$
F_{2}\left(W, Q^{2}\right)=\frac{Q^{2}}{4 \pi^{2} \alpha_{\mathrm{em}}}\left[\sigma_{T}\left(W, Q^{2}\right)+\sigma_{L}\left(W, Q^{2}\right)\right]
$$

up to terms of order $\mathcal{O}\left(Q^{2} / W^{2}\right)$. Similarly, we use for the standard longitudinal structure function

$$
F_{L}\left(W, Q^{2}\right)=\frac{Q^{2}}{4 \pi^{2} \alpha_{\mathrm{em}}} \sigma_{L}\left(W, Q^{2}\right) .
$$

In the following we shall use the relation (2.14) valid in the high energy limit. In appendix $\mathrm{A}$ we shall discuss how our results are modified for finite Bjorken- $x$ if we use the exact formula (2.13) instead of (2.14). We note that one could also consider (2.14) as the defining equation for $\sigma_{T}$ and $\sigma_{L}$. This would correspond to a different choice of flux factor for the virtual photons as compared to 26 . The considerations of section 6 of [23] show, however, that Hand's convention [26] is the natural one for the dipole picture; see especially (121)-(128) of [23].

In [22, 23] nonperturbative methods were employed in order to work towards a foundation of the dipole model for quasi-real and virtual photon induced reactions at high energies. The result for $W^{\mu \nu}$ (2.5) obtained there is shown diagrammatically in Fig. 1. In the high energy limit, $q^{0} \rightarrow \infty$, taken in the proton rest frame, we find a factorization into photon wave function and dipoleproton scattering parts. The wave function parts contain the renormalized $\gamma q \bar{q}$ vertex function plus a rescattering term. The dipole-proton scattering is built from diagrams of type (a) where the quark lines go through from right to left and type (b) where the quark lines do not go through. To get from there to the standard dipole picture requires to make a number of assumptions and approximations as listed in [23]:

(i) Quarks of flavor $q$ have a mass shell $m_{q}$ and can be considered as asymptotic states.

(ii) The rescattering terms are dropped and the $\gamma q \bar{q}$ vertex functions are replaced by the lowest order terms in perturbation theory. (iii) The $T$-matrix element for the dipole-proton scattering is diagonal in the quark flavor $q$, in $\alpha$ and in $\boldsymbol{r}$. Here $\alpha$ is the longitudinal momentum fraction of the photon carried by the quark, and $\boldsymbol{r}$ is the two-dimensional vector from the antiquark to the quark in transverse position space. Further, the $T$ matrix element is proportional to the unit matrix in the space of spin orientations of the quark and antiquark in the dipole.

(iv) In the $T$-matrix element for the dipole-proton scattering only the contribution of type (a) is kept while that of type (b) is neglected, see Fig. 1.

(v) The proton spin averaged reduced matrix element for a given quark flavor $q$ depends only on the dipole size $r \equiv \sqrt{\boldsymbol{r}^{2}}$ and on $W^{2}=(p+q)^{2}$.

With these assumptions we arrive indeed at the standard formulae of the dipole picture used extensively in the literature. The squared and spin-summed photon wave functions for quark flavor $q$ can be calculated in leading order in $\alpha_{\mathrm{em}}$ resulting in

$$
\begin{gathered}
\sum_{\lambda, \lambda^{\prime}}\left|\psi_{\gamma, \lambda \lambda^{\prime}}^{(q) \mu}(\alpha, \boldsymbol{r}, Q) \varepsilon_{+\mu}\right|^{2} \\
=\frac{N_{c}}{2 \pi^{2}} \alpha_{\mathrm{em}} Q_{q}^{2}\left\{\left[\alpha^{2}+(1-\alpha)^{2}\right] \epsilon_{q}^{2}\left[K_{1}\left(\epsilon_{q} r\right)\right]^{2}\right. \\
\left.+m_{q}^{2}\left[K_{0}\left(\epsilon_{q} r\right)\right]^{2}\right\} \\
\sum_{\lambda, \lambda^{\prime}}\left|\psi_{\gamma, \lambda \lambda^{\prime}}^{(q) \mu}(\alpha, \boldsymbol{r}, Q) \varepsilon_{L \mu}^{\prime}\right|^{2} \\
=\frac{2 N_{c}}{\pi^{2}} \alpha_{\mathrm{em}} Q_{q}^{2} Q^{2}[\alpha(1-\alpha)]^{2}\left[K_{0}\left(\epsilon_{q} r\right)\right]^{2}
\end{gathered}
$$

for transversely and longitudinally polarized photons, respectively. Here $N_{c}=3$ is the number of colors, $\epsilon_{q}=\sqrt{\alpha(1-\alpha) Q^{2}+m_{q}^{2}}, Q_{q}$ denotes the quark charges in units of the proton charge, and $K_{0,1}$ are modified Bessel functions. Upon integration over $\alpha$ we obtain from the above expressions the photon densities as functions of the dipole size $r$ and of $Q^{2}$,

$$
\begin{aligned}
& w_{T}^{(q)}\left(r, Q^{2}\right)=\sum_{\lambda, \lambda^{\prime}} \int_{0}^{1} \mathrm{~d} \alpha\left|\psi_{\gamma, \lambda \lambda^{\prime}}^{(q) \mu}(\alpha, \boldsymbol{r}, Q) \varepsilon_{+\mu}\right|^{2}, \\
& w_{L}^{(q)}\left(r, Q^{2}\right)=\sum_{\lambda, \lambda^{\prime}} \int_{0}^{1} \mathrm{~d} \alpha\left|\psi_{\gamma, \lambda \lambda^{\prime}}^{(q) \mu}(\alpha, \boldsymbol{r}, Q) \varepsilon_{L \mu}^{\prime}\right|^{2} .
\end{aligned}
$$

The expressions for the $\gamma^{(*)} p$ total cross sections in the standard dipole picture are

$$
\begin{aligned}
& \sigma_{T}\left(W, Q^{2}\right)=\sum_{q} \int \mathrm{d}^{2} r w_{T}^{(q)}\left(r, Q^{2}\right) \hat{\sigma}^{(q)}(r, W), \\
& \sigma_{L}\left(W, Q^{2}\right)=\sum_{q} \int \mathrm{d}^{2} r w_{L}^{(q)}\left(r, Q^{2}\right) \hat{\sigma}^{(q)}(r, W) .
\end{aligned}
$$


(a)
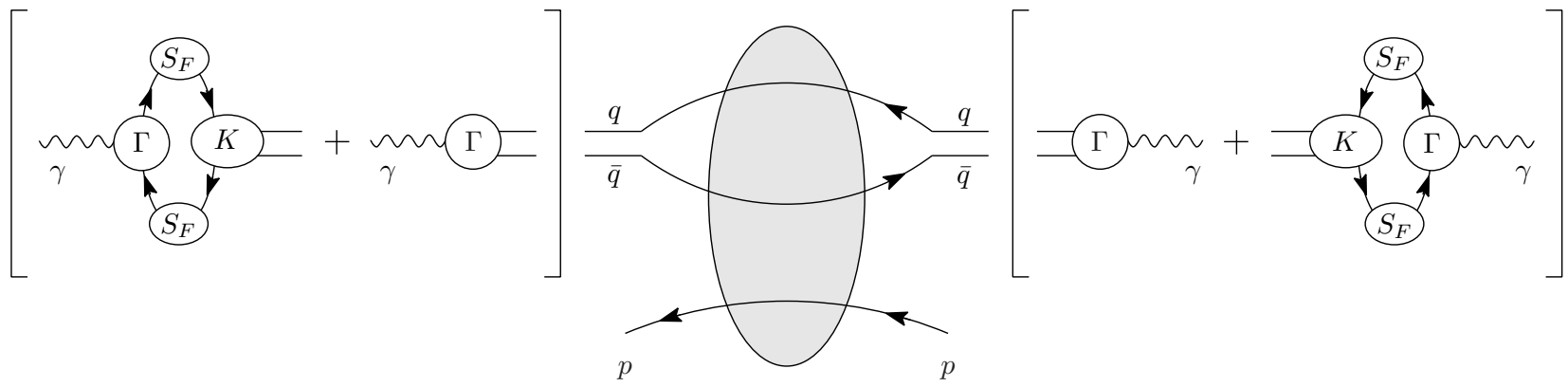

(b)

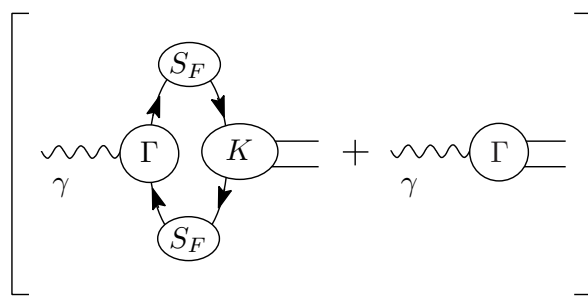

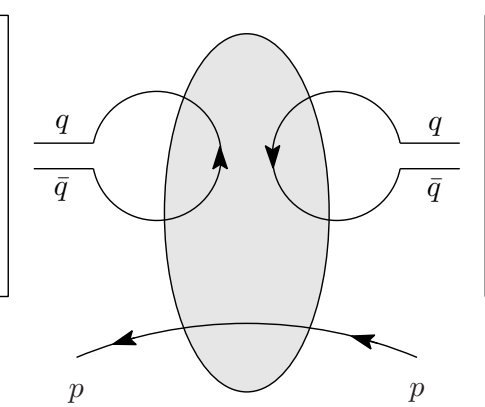

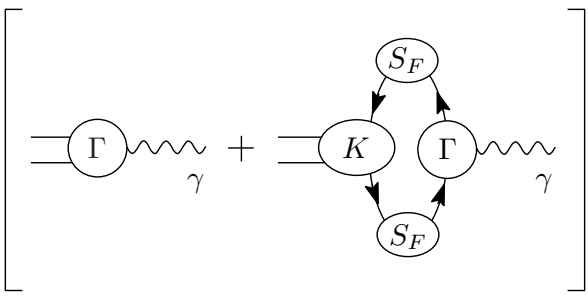

FIG. 1: Quark skeleton diagrams for the photon-proton scattering cross section in the high energy limit. The shaded area indicates a functional integration over gluon field configurations, and $\Gamma, K, S_{F}$ are the renormalized $\gamma q \bar{q}$ vertex, the renormalized kernel for $q^{\prime} \bar{q}^{\prime}$ to $q \bar{q}$ scattering, and the renormalized quark propagator, respectively. The diagrams are to be read from right to left.

Here $\hat{\sigma}^{(q)}(r, W)$ is the cross section for the scattering of a dipole of flavor $q$ and size $r$ on a proton for a dipoleproton c.m. energy $W$. Presently, the dipole-proton cross section cannot be calculated from first principles and one therefore uses models with parameters obtained by fitting the available data.

Note that the correct energy variable of the dipoleproton cross section $\hat{\sigma}$ is $W$ and not Bjorken- $x$. The latter would imply a dependence on the photon virtuality $Q^{2}$. It was argued in 23] that using $x$ instead of $W$ requires additional assumptions which are difficult to assess quantitatively and which go beyond those listed above. Nevertheless, the energy variable $x$ - and hence a $Q^{2}$-dependence - is frequently used in popular models for the dipole cross section (and sometimes other dependencies on $Q^{2}$ are introduced).

In the following we shall also consider $F_{2}^{(c)}$, that is, the structure function for $\gamma^{*} p$ scattering with production of charm particles. In the dipole model the cross sections for charm production are obtained as in (2.20), (2.21) but without the summation over quark flavor $q$,

$$
\sigma_{T, L}^{(c)}\left(W, Q^{2}\right)=\int \mathrm{d}^{2} r w_{T, L}^{(c)}\left(r, Q^{2}\right) \hat{\sigma}^{(c)}(r, W) .
$$

We set as in (2.14) for the charm structure function

$$
F_{2}^{(c)}=\frac{Q^{2}}{4 \pi^{2} \alpha_{\mathrm{em}}}\left[\sigma_{T}^{(c)}\left(W, Q^{2}\right)+\sigma_{L}^{(c)}\left(W, Q^{2}\right)\right] .
$$

Note that in doing so we make the assumption that all charm quarks produced originate directly from the initial $\gamma^{*}$. That is, we neglect associated charm-anticharm production in reactions initiated by other quark flavors coupling directly to the photon.

In the following sections we shall use (2.20)-(2.22) to derive bounds for ratios of structure functions. These bounds will rest on the explicit forms of the photon densities $w_{T, L}^{(q)}(2.18),(2.19)$ and on the non-negativity of the dipole-proton cross sections

$$
\hat{\sigma}^{(q)}(r, W) \geq 0 .
$$

The bounds derived in Sect. III remain unchanged if we assume that the dipole cross sections $\hat{\sigma}^{(q)}$ are functions of $r$ and Bjorken- $x$ instead of $r$ and $W$. The bounds derived in Sect. IV] on the other hand, depend crucially on the functional dependence indicated in (2.24).

\section{BOUNDS FOR $F_{L} / F_{2}$ AND $F_{2}^{(c)} / F_{2}$}

In this section we consider the structure functions $F_{L}$, $F_{2}^{(c)}$, and $F_{2}$ at fixed values of $Q^{2}$ and $W$. Arranging them into a three-vector gives according to the dipole formula

$$
\left(\begin{array}{c}
F_{L}\left(W, Q^{2}\right) \\
F_{2}^{(c)}\left(W, Q^{2}\right) \\
F_{2}\left(W, Q^{2}\right)
\end{array}\right)=\sum_{q} \int \mathrm{d}^{2} r \frac{\hat{\sigma}^{(q)}(r, W)}{4 \pi^{2} \alpha_{\mathrm{em}}}\left(\begin{array}{r}
f_{L}^{(q)}\left(r, Q^{2}\right) \\
\delta_{q, c} f^{(c)}\left(r, Q^{2}\right) \\
f^{(q)}\left(r, Q^{2}\right)
\end{array}\right),
$$


with

$$
\begin{aligned}
& f^{(q)}\left(r, Q^{2}\right)=Q^{2}\left[w_{T}^{(q)}\left(r, Q^{2}\right)+w_{L}^{(q)}\left(r, Q^{2}\right)\right], \\
& f_{L}^{(q)}\left(r, Q^{2}\right)=Q^{2} w_{L}^{(q)}\left(r, Q^{2}\right) .
\end{aligned}
$$

Note that the second entry in the vector in (3.1) receives a contribution only from the charm quark, as indicated by the Kronecker delta symbol. In the following we will make use of a geometrical interpretation of (3.1) in order to obtain correlated bounds on the structure functions involved here. Due to the Kronecker symbol the case at hand is somewhat special, which might make the geometrical interpretation slightly more difficult to conceive. An illustration of the general argument is given in Fig. 4 in section [IV] below where we discuss similar three-vectors of structure functions, but there without the occurrence of a Kronecker symbol.

We recall that the dipole cross sections $\hat{\sigma}^{(q)}$ are nonnegative. Thus the r.h.s. of (3.1) is a sum and an integral over three-vectors multiplied by non-negative weights, or, in other words, a special linear superposition of the threevectors appearing under the integral. We want to find the set of all possible linear superpositions of this kind with non-negative coefficients. This is called a moment problem. In appendix B we discuss the necessary mathematical tools to solve this problem. We give there the precise definitions of the key concepts convex set, convex hull and convex cone. We also give the detailed solution of the moment problem for the case of three $F_{2}^{(q)}$ structure functions as discussed below in section [IV] The solution of the moment problem in this section runs along the same lines. The analogue of the result (B49) reads here as follows. The set of all vectors allowing a representation (3.1) is given by a convex cone. Any vector within this cone can be written as a non-negative multiple of an element within the closed convex hull (denoted by $\overline{\mathrm{co}}$ ) of the three-vectors appearing in the r.h.s. of (3.1). Therefore we have

$$
\left(\begin{array}{c}
F_{L}\left(W, Q^{2}\right) \\
F_{2}^{(c)}\left(W, Q^{2}\right) \\
F_{2}\left(W, Q^{2}\right)
\end{array}\right)=\lambda \boldsymbol{u}\left(Q^{2}\right), \quad \lambda \geq 0, \quad \boldsymbol{u}\left(Q^{2}\right) \in \overline{\operatorname{co}}\left\{\left(\begin{array}{r}
f_{L}^{(q)}\left(r, Q^{2}\right) \\
\delta_{q, c} f^{(c)}\left(r, Q^{2}\right) \\
f^{(q)}\left(r, Q^{2}\right)
\end{array}\right) \mid r \in \mathbb{R}^{+}, q=u, d, \ldots\right\}
$$

Note that the three-vectors from which the convex hull is constructed involve only the functions $f^{(q)}\left(r, Q^{2}\right)$ and $f_{L}^{(q)}\left(r, Q^{2}\right)$ which are for any given $Q^{2}$ explicitly known for all $r$, see (2.16)-(2.19). Hence it is also straightforward to compute their convex hull. We further point out that these vectors are independent of the energy $W$, and that the condition (3.4) does not involve any model assumption about the dipole cross section $\hat{\sigma}^{(q)}$.

We can now use the condition (3.4) to derive bounds on ratios of $F_{L}, F_{2}^{(c)}$, and $F_{2}$. These bounds originate only from the photon wave functions. They will be valid for any dipole cross section $\hat{\sigma}^{(q)}$, and will be independent of the energy $W$. Clearly, the bounds will vary with the photon virtuality $Q^{2}$, since $Q^{2}$ explicitly enters the vectors in (3.4) via the photon wave function.

We first notice that the condition (3.4) constrains only the directions of the three-vectors involved, while their normalization is irrelevant for that condition. We can therefore normalize the vector composed of the three structure functions such that its third component equals one, that is, we consider the vector $\left(F_{L} / F_{2}, F_{2}^{(c)} / F_{2}, 1\right)^{\mathrm{T}}$ instead of $\left(F_{L}, F_{2}^{(c)}, F_{2}\right)^{\mathrm{T}}$. That normalization does not change the direction of the vector, and hence also the so normalized vector fulfills the condition (3.4). Similarly, we can also normalize the set of vectors of which the closed convex hull is formed such that its third component equals one, hence consider$\operatorname{ing}\left(f_{L}^{(q)} / f^{(q)}, \delta_{q, c} f^{(c)} / f^{(q)}, 1\right)^{\mathrm{T}}=\left(f_{L}^{(q)} / f^{(q)}, \delta_{q, c}, 1\right)^{\mathrm{T}}$ in place of $\left(f_{L}^{(q)}, \delta_{q, c} f^{(c)}, f^{(q)}\right)^{\mathrm{T}}$. Again, that does not affect the direction of the vectors, and the condition (3.4) immediately applies with this replacement. The condition with both vectors normalized in this way contains only vectors the third component of which equals one, and for this case the only possible choice for the factor $\lambda$ is $\lambda=1$. We can then eliminate the trivial third component by projecting onto the 1-2-plane and obtain from (3.4) the simpler condition

$$
\left(\begin{array}{c}
F_{L}\left(W, Q^{2}\right) / F_{2}\left(W, Q^{2}\right) \\
F_{2}^{(c)}\left(W, Q^{2}\right) / F_{2}\left(W, Q^{2}\right)
\end{array}\right) \in \overline{\mathrm{co}}\left\{\left(\begin{array}{c}
f_{L}^{(q)}\left(r, Q^{2}\right) / f^{(q)}\left(r, Q^{2}\right) \\
\delta_{q, c}
\end{array}\right) \mid r \in \mathbb{R}^{+}, q=u, d, \ldots\right\}
$$




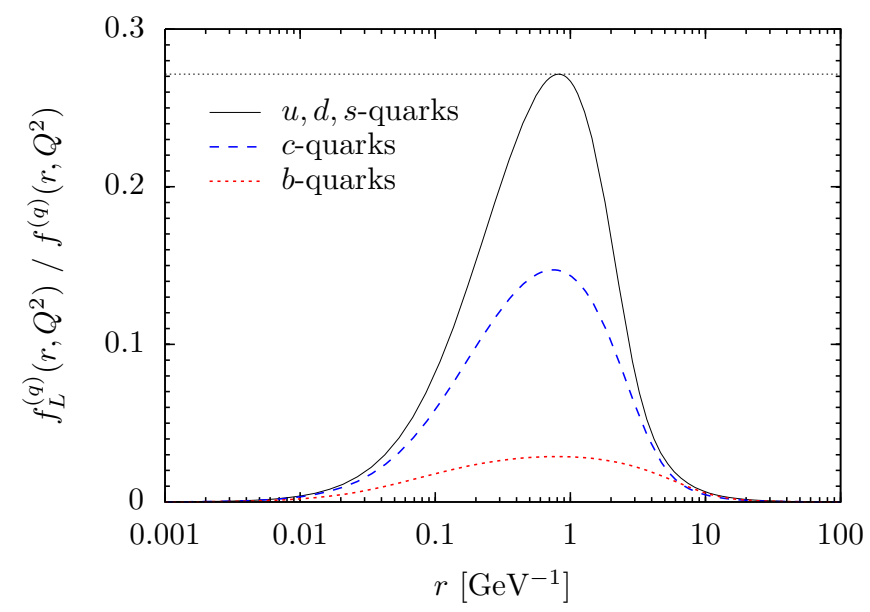

FIG. 2: The ratio $f_{L}^{(q)}\left(r, Q^{2}\right) / f^{(q)}\left(r, Q^{2}\right)$ as a function of $r$ for different quark flavors. The photon virtuality is chosen to be $Q^{2}=10 \mathrm{GeV}^{2}$. The absolute maximum value (dotted line) of all curves provides an upper bound on $F_{L}\left(W, Q^{2}\right) / F_{2}\left(W, Q^{2}\right)$, see (3.6) and (3.7).

strictly positive. For a rigorous derivation of bounds on ratio vectors as in (3.5) see appendix B, where the analogous case of three $F_{2}$ structure functions is discussed in detail (cf. (B52)).

The first bound that we want to discuss here is now obtained from (3.5) by projecting onto the 1-axis. This immediately gives

$$
\inf _{r, q} \frac{f_{L}^{(q)}\left(r, Q^{2}\right)}{f^{(q)}\left(r, Q^{2}\right)} \leq \frac{F_{L}\left(W, Q^{2}\right)}{F_{2}\left(W, Q^{2}\right)} \leq \sup _{r, q} \frac{f_{L}^{(q)}\left(r, Q^{2}\right)}{f^{(q)}\left(r, Q^{2}\right)}
$$

where inf and sup denote the infimum and supremum, respectively [35]. Note that these lower and upper bounds on $F_{L} / F_{2}$ are given only in terms of the photon wave function. It is therefore straightforward to analyze the bounds (3.6) numerically.

In Fig. 2 we plot the ratio $f_{L}^{(q)}\left(r, Q^{2}\right) / f^{(q)}\left(r, Q^{2}\right)$ as a function of $r$ for different quark flavors, choosing as an example $Q^{2}=10 \mathrm{GeV}^{2}$. Here and in the following we use vanishing masses for the light $(u, d, s)$ quarks, $m_{c}=$ $1.3 \mathrm{GeV}$ for the charm quark and $m_{b}=4.6 \mathrm{GeV}$ for the bottom quark. We find that the lower bound in 3.6 is trivial, $F_{L} / F_{2} \geq 0$. The upper bound, on the other hand, is nontrivial, and we find that the maximal value of $f_{L}^{(q)}\left(r, Q^{2}\right) / f^{(q)}\left(r, Q^{2}\right)$ is obtained for light quarks, as can be seen in Fig. 2 where this maximum is drawn as a dotted horizontal line. It turns out that this upper bound is independent of $Q^{2}$ and numerically leads to

$$
\frac{F_{L}\left(W, Q^{2}\right)}{F_{2}\left(W, Q^{2}\right)} \leq 0.27139
$$

A stronger bound can be obtained by considering the correlation of the ratios $F_{L} / F_{2}$ and $F_{2}^{(c)} / F_{2}$, that is, by taking into account both components of the constraint on the vectors in (3.5). In this case the bound on the ratio
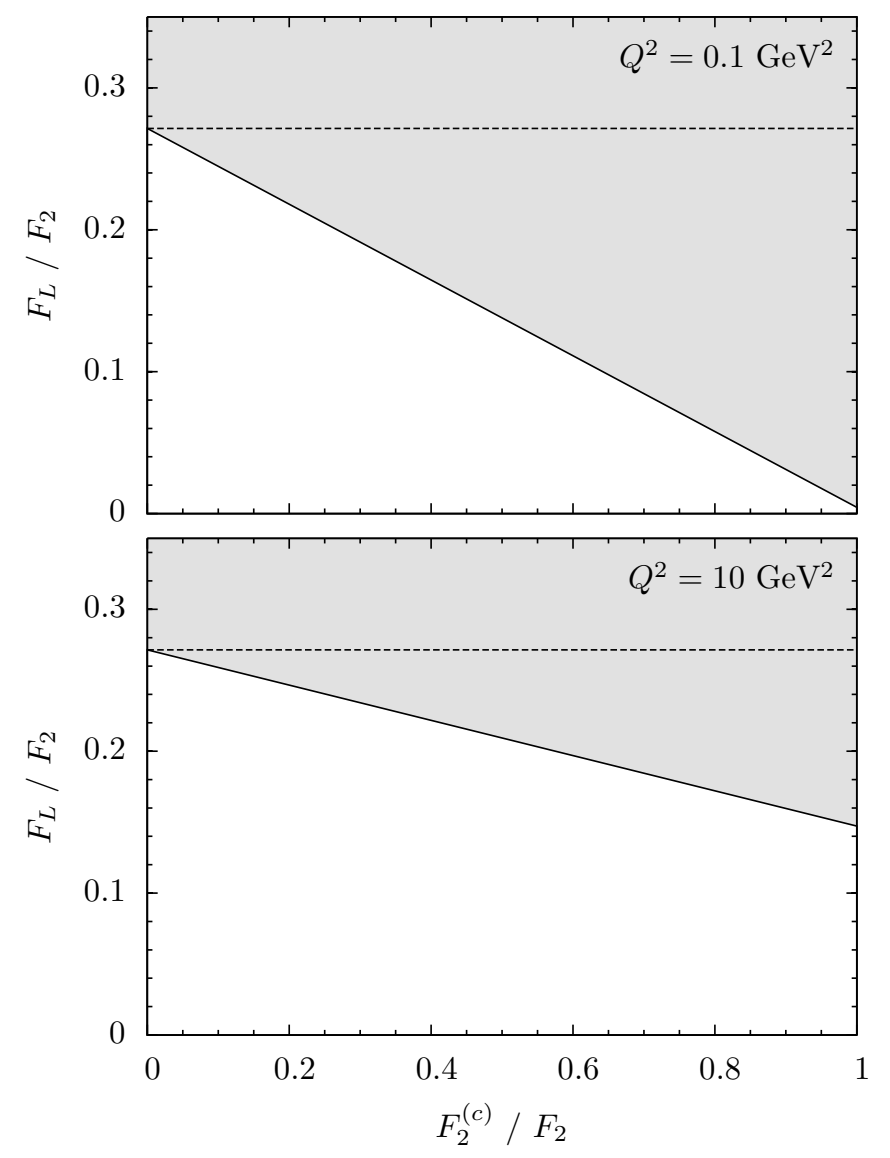

FIG. 3: Correlated bounds on $F_{L} / F_{2}$ and $F_{2}^{(c)} / F_{2}$. Only the white (unshaded) area is allowed within the dipole picture. The weaker bound (3.6) is shown as a dashed line, while the stronger bound implied by (3.5) is shown as a solid line.

$F_{L} / F_{2}$ will depend on the value of $F_{2}^{(c)} / F_{2}$ or vice versa. By computing the closed convex hull in (3.5) from the ratios $f_{L}^{(q)}\left(W, Q^{2}\right) / f^{(q)}\left(W, Q^{2}\right)$ we obtain the correlated bounds shown in Fig. 3 for the two values $Q^{2}=0.1 \mathrm{GeV}^{2}$ and $Q^{2}=10 \mathrm{GeV}^{2}$. The unshaded area in the two plots is the allowed region in the dipole picture. The Figure is drawn for the whole range of $F_{2}^{(c)} / F_{2}$ between zero and one to make the origin of the bounds clear. Realistic values of $F_{2}^{(c)} / F_{2}$ can only range from zero to at most about 0.4. The allowed area in Fig. 3 is bounded by a straight line. This particular shape emerges due to the fact that the second component of the vectors in (3.5) receives a contribution only from the charm quark. Due to the corresponding Kronecker symbol the upper bound on $F_{L} / F_{2}$ at the (unphysical) point $F_{2}^{(c)} / F_{2}=1$ is given by the maximum of $f_{L}^{(c)}\left(r, Q^{2}\right) / f^{(c)}\left(r, Q^{2}\right)$ over all $r$ for the $Q^{2}$ under consideration. The value of this maximum for the case $Q^{2}=10 \mathrm{GeV}^{2}$ can be read off from the charm quark curve in Fig. 2, For $Q^{2}$-values well below the charm mass, like for example $Q^{2}=0.1 \mathrm{GeV}^{2}$, the analogous function practically vanishes and the resulting 
upper bound on $F_{L} / F_{2}$ at $F_{2}^{(c)} / F_{2}=1$ is practically zero. The fact that the unphysical point $F_{2}^{(c)} / F_{2}=1$ is relevant for the determination of the correlated bounds on $F_{L} / F_{2}$ and $F_{2}^{(c)} / F_{2}$ in the physical region of these ratios should not cause any worries here. It is just the consequence of not making any assumptions about the flavor dependence of the dipole cross sections $\hat{\sigma}^{(q)}$, except their being non-negative. This general case includes for example the unphysical case that all dipole cross sections but the one for the charm quark would vanish, which would give rise to $F_{2}^{(c)} / F_{2}=1$. By making further assumptions about the dipole cross sections it should be possible to derive more stringent bounds - but at the expense of introducing a dependence on those assumptions. In the present paper, however, it is our aim to study bounds on ratios of structure functions from the dipole picture which do not depend on any further assumptions on the dipole cross section.

Future measurements of the structure functions $F_{L}$, $F_{2}^{(c)}$ and $F_{2}$ at identical values of $Q^{2}$ and $W$ might in combination with our bounds be able to constrain the range of validity of the dipole picture.

Closing this section we would like to point out again that the geometric argument and its implications discussed in this section remain unchanged if the dipole cross section is chosen to depend on $x$ instead of $W$.

\section{BOUNDS ON RATIOS OF $F_{2}$ AT DIFFERENT VALUES OF $Q^{2}$}

In this section we use the dipole picture to derive bounds on ratios of the structure function $F_{2}$ taken at the same $W$ but at different values of $Q^{2}$. The results found here crucially depend on choosing the functional dependence of the dipole cross section such that its arguments are $r$ and $W$. In particular, the dipole cross section $\hat{\sigma}^{(q)}(r, W)$ is assumed to be independent of $Q^{2}$, see the corresponding discussion in section II.

We consider the structure function $F_{2}$ at three different values of $Q^{2}$ but at the same $W$. Similarly to the previous section we arrange them into a three-vector, and evaluate it according to the dipole formula,

$$
\left(\begin{array}{l}
F_{2}\left(W, Q_{1}^{2}\right) \\
F_{2}\left(W, Q_{2}^{2}\right) \\
F_{2}\left(W, Q_{3}^{2}\right)
\end{array}\right)=\sum_{q} \int \mathrm{d}^{2} r \frac{\hat{\sigma}^{(q)}(r, W)}{4 \pi^{2} \alpha_{\mathrm{em}}}\left(\begin{array}{l}
f^{(q)}\left(r, Q_{1}^{2}\right) \\
f^{(q)}\left(r, Q_{2}^{2}\right) \\
f^{(q)}\left(r, Q_{3}^{2}\right)
\end{array}\right)
$$

where the $f^{(q)}\left(r, Q_{i}^{2}\right)$ are defined in (3.2). We can now derive bounds on ratios of such structure functions following the same procedure as in the preceeding section. To find all vectors allowing a representation (4.1) is again a moment problem. In appendix B we discuss the solution of this problem for the case at hand in a mathematically rigorous way. A simple argument, leaving out some subtleties, is as follows.

The vector on the l.h.s. of (4.1) is a linear superposition of the vectors $\left(f^{(q)}\left(r, Q_{1}^{2}\right), f^{(q)}\left(r, Q_{2}^{2}\right), f^{(q)}\left(r, Q_{3}^{2}\right)\right)^{\mathrm{T}}$ which appear under the integral. For a given flavor $q$ and given values of the $Q_{i}^{2}$ that vector follows a trajectory as $r \in \mathbb{R}^{+}$is varied. Fig. (4 illustrates a number of vectors along such a trajectory for the case of massless quarks and for one particular choice of $Q_{1}^{2}, Q_{2}^{2}$, and $Q_{3}^{2}$. We

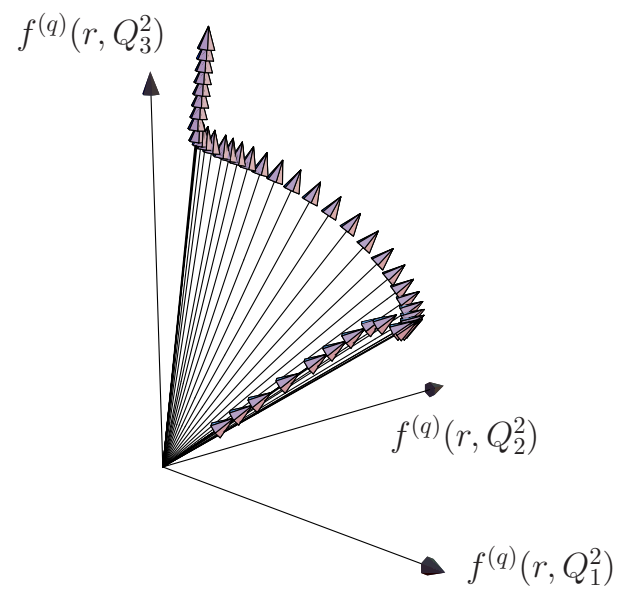

FIG. 4: The vectors $\left(f^{(q)}\left(r, Q_{1}^{2}\right), f^{(q)}\left(r, Q_{2}^{2}\right), f^{(q)}\left(r, Q_{3}^{2}\right)\right)^{\mathrm{T}}$ for different values of $r$, shown here for a massless quark flavor $q$ and for one particular choice of the triple $\left(Q_{1}^{2}, Q_{2}^{2}, Q_{3}^{2}\right)$.

recall again that the dipole cross sections $\hat{\sigma}^{(q)}$ are nonnegative. Accordingly, the r.h.s. of (4.1) is a linear superposition with non-negative weights of the vectors that appear under the integral. Therefore the resulting vector on the l.h.s. must lie in the closed convex cone formed by all possible linear superpositions with non-negative weights of those vectors and their boundary. Any vector within such a cone is a non-negative multiple of a vector that lies in the closed convex hull (denoted by $\overline{\mathrm{co}}$ ) of the vectors appearing under the integral in (4.1), see (B49) of appendix B. Hence we obtain the condition

$$
\left(\begin{array}{l}
F_{2}\left(W, Q_{1}^{2}\right) \\
F_{2}\left(W, Q_{2}^{2}\right) \\
F_{2}\left(W, Q_{3}^{2}\right)
\end{array}\right)=\mu \boldsymbol{v}\left(Q_{1}^{2}, Q_{2}^{2}, Q_{3}^{2}\right), \quad \mu \geq 0, \quad \boldsymbol{v}\left(Q_{1}^{2}, Q_{2}^{2}, Q_{3}^{2}\right) \in \overline{\mathrm{co}}\left\{\left(\begin{array}{l}
f^{(q)}\left(r, Q_{1}^{2}\right) \\
f^{(q)}\left(r, Q_{2}^{2}\right) \\
f^{(q)}\left(r, Q_{3}^{2}\right)
\end{array}\right) \mid r \in \mathbb{R}^{+}, q=u, d, \ldots\right\}
$$


but not their length. This applies both to the vector with components $F_{2}\left(W, Q_{i}^{2}\right)$ and to the vector with components $f^{(q)}\left(r, Q_{i}^{2}\right)$. Accordingly, we can normalize these vectors such that their third component equals one. Doing this for both vectors that appear in (4.2) we obtain an equivalent condition. In this condition the only possible value for $\mu$ is obviously $\mu=1$. Since the third component of the condition is now trivial we discard it by projecting onto the 1-2-plane to obtain

$$
\left(\begin{array}{l}
F_{2}\left(W, Q_{1}^{2}\right) / F_{2}\left(W, Q_{3}^{2}\right) \\
F_{2}\left(W, Q_{2}^{2}\right) / F_{2}\left(W, Q_{3}^{2}\right)
\end{array}\right) \in \overline{\operatorname{co}}\left\{\left(\begin{array}{c}
f^{(q)}\left(r, Q_{1}^{2}\right) / f^{(q)}\left(r, Q_{3}^{2}\right) \\
f^{(q)}\left(r, Q_{2}^{2}\right) / f^{(q)}\left(r, Q_{3}^{2}\right)
\end{array}\right) \mid r \in \mathbb{R}^{+}, q=u, d, \ldots\right\}
$$

which is fully equivalent to (4.2) because $F_{2}\left(W, Q_{3}^{2}\right)$ and $f^{(q)}\left(r, Q_{3}^{2}\right)$ are strictly positive for the relevant range of their arguments. For a rigorous derivation of (4.3) see (B50)-(B52) of appendix B.

Let us first consider the two components of the condition (4.3) separately. Projecting it onto the 1-axis and onto the 2-axis immediately gives the conditions

$$
\begin{aligned}
& \inf _{r, q} \frac{f^{(q)}\left(r, Q_{1}^{2}\right)}{f^{(q)}\left(r, Q_{3}^{2}\right)} \leq \frac{F_{2}\left(W, Q_{1}^{2}\right)}{F_{2}\left(W, Q_{3}^{2}\right)} \leq \sup _{r, q} \frac{f^{(q)}\left(r, Q_{1}^{2}\right)}{f^{(q)}\left(r, Q_{3}^{2}\right)}, \\
& \inf _{r, q} \frac{f^{(q)}\left(r, Q_{2}^{2}\right)}{f^{(q)}\left(r, Q_{3}^{2}\right)} \leq \frac{F_{2}\left(W, Q_{2}^{2}\right)}{F_{2}\left(W, Q_{3}^{2}\right)} \leq \sup _{r, q} \frac{f^{(q)}\left(r, Q_{2}^{2}\right)}{f^{(q)}\left(r, Q_{3}^{2}\right)},
\end{aligned}
$$

respectively. The condition (4.5) goes into (4.4) if we replace $Q_{2}^{2}$ by $Q_{1}^{2}$. Thus, for two given values of $Q^{2}$ we actually obtain one condition here which contains an upper and and a lower bound. The same result was already presented in 24], where these bounds were derived in a different way.

We find it useful to discuss here briefly the bounds (4.4), for a more detailed discussion we refer the reader to 24]. We first note that the upper and lower bound (4.4) depend only on the values of $Q_{1}^{2}$ and $Q_{3}^{2}$, but do not involve the energy $W$. Fig. 5 shows the ratio $f^{(q)}\left(r, Q_{1}^{2}\right) / f^{(q)}\left(r, Q_{3}^{2}\right)$ as a function of $r$ for different quark masses along with the resulting bounds on $F_{2}\left(W, Q_{1}^{2}\right) / F_{2}\left(W, Q_{3}^{2}\right)$ for a concrete choice of $Q_{1}^{2}, Q_{3}^{2}$, $\left(Q_{1}^{2}, Q_{3}^{2}\right)=(2,10) \mathrm{GeV}^{2}$.

In the following we want to confront the bounds that we obtain in this section from the color dipole picture with HERA data. Before we proceed with that a remark is in order concerning that comparison with data. Data on $F_{2}$ and measurements of the reduced cross section are available for a large range of $Q^{2}$ values with $\left(x, Q^{2}\right)$-binning. However, throughout this section we deal with bounds involving values of $F_{2}$ (or of the reduced cross section, see below) at the same $W$ but at different $Q_{i}^{2}$. Hence a comparison with our bounds requires different values of $Q^{2}$ at the same value of $W$, and data with $\left(W, Q^{2}\right)$-binning are published only for comparatively small kinematical ranges. We therefore use a fit to the $F_{2}$ data that can, to a good approximation, be considered as a substitute of actual data. We do this in most of the following comparisons, except for two illustrations where HERA data are used directly (see Fig. 8

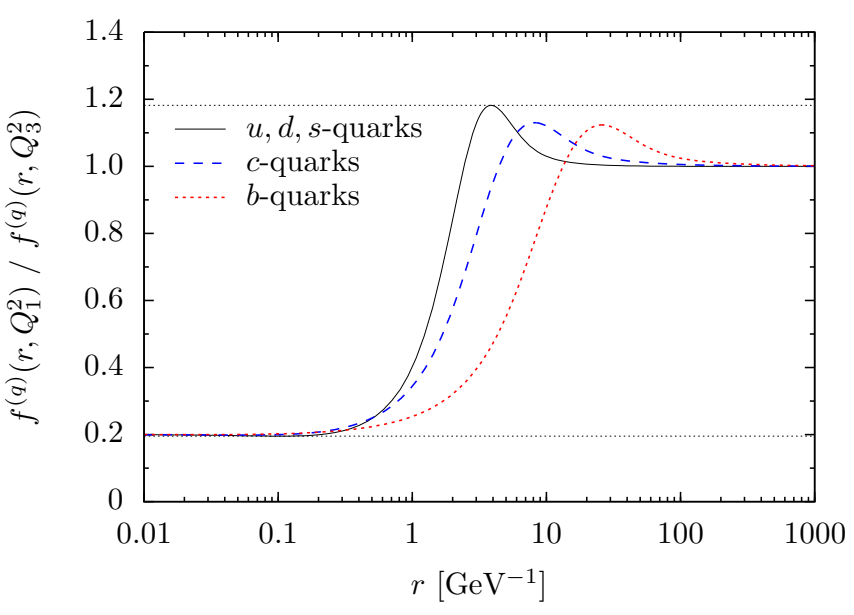

FIG. 5: The ratio $f^{(q)}\left(r, Q_{1}^{2}\right) / f^{(q)}\left(r, Q_{3}^{2}\right)$ as a function of $r$ for different quark masses, here for the choice $\left(Q_{1}^{2}, Q_{3}^{2}\right)=$ $(2,10) \mathrm{GeV}^{2}$. The minimum and maximum values of all curves (shown as dotted lines) provide a lower and upper bound on $F_{2}\left(W, Q_{1}^{2}\right) / F_{2}\left(W, Q_{3}^{2}\right)$, respectively, see (4.4).

and the corresponding discussion below). Concretely, we use the ALLM97 fit to $F_{2}$ [27, 28] which represents the measured data points of [1] - [5] within their errors, except maybe for the region of very low $Q^{2}$ where the fit appears to be slightly worse. We emphasize that we use the fit only inside the kinematical range in which actual HERA data are available. No extrapolation beyond that range is done here.

Fig. 6] confronts the bound (4.4) with the ALLM97 fit to $F_{2}$ for a fixed value of $Q_{3}^{2}$ and variation of $Q_{1}^{2}$, as presented in 24] before. It is apparent from the Figure that there is a value of $Q_{1}^{2}$ beyond which the dipole picture fails to be compatible with the ALLM97 fit. This maximal $Q_{1}^{2}$ value depends on the value of $W$, as can be seen in the Figure from the three curves for different $W$, and it also depends on the value chosen for $Q_{3}^{2}$. With the choice $Q_{3}^{2}=10 \mathrm{GeV}^{2}$ made for the Figure this maximal $Q_{1}^{2}$ is in the range of about $150-300 \mathrm{GeV}^{2}$, depending on $W$.

So far we have discussed in some detail the bounds (4.4) and 4.5) which resulted from considering the two components of (4.3) separately. We can im- 


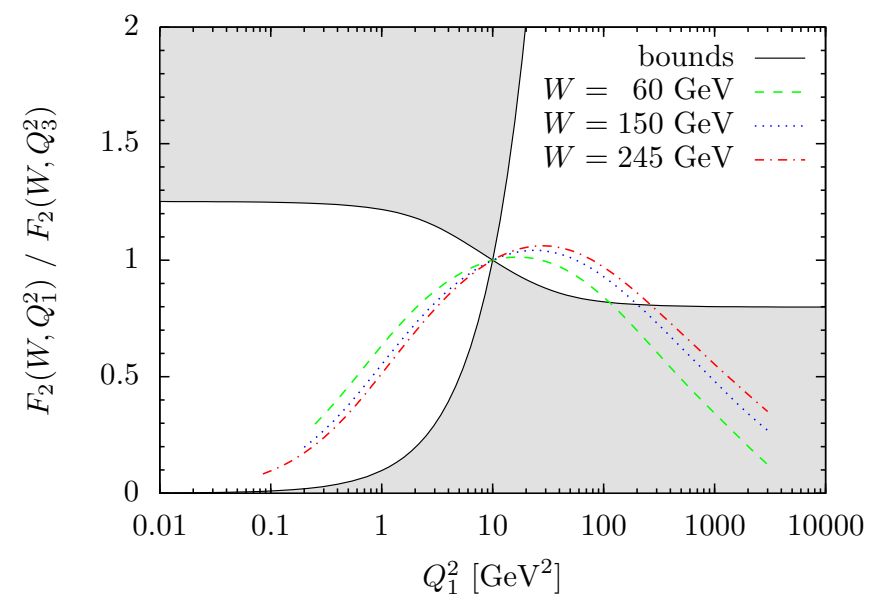

FIG. 6: The bounds (4.4) on $F_{2}\left(W, Q_{1}^{2}\right) / F_{2}\left(W, Q_{3}^{2}\right)$ resulting from the dipole picture (solid lines) confronted with the corresponding ratios obtained using the ALLM97 fit to $F_{2}$ for three different values of $W$. Here $Q_{1}^{2}$ is varied while the value $Q_{3}^{2}=10 \mathrm{GeV}^{2}$ is kept fixed. The shaded region is excluded by the bounds.

prove these bounds by taking into account the correlation of those two components, that is the correlation of the two ratios $F_{2}\left(W, Q_{1}^{2}\right) / F_{2}\left(W, Q_{3}^{2}\right)$ and $F_{2}\left(W, Q_{2}^{2}\right) / F_{2}\left(W, Q_{3}^{2}\right)$. According to (4.3) the 2-vector constructed of these two ratios for a given set of $Q_{i}^{2}$ lies in the closed convex hull of the vectors $\left(f^{(q)}\left(r, Q_{1}^{2}\right) / f^{(q)}\left(r, Q_{3}^{2}\right), f^{(q)}\left(r, Q_{2}^{2}\right) / f^{(q)}\left(r, Q_{3}^{2}\right)\right)^{\mathrm{T}}$. As $r$ is varied the latter vector (for each quark flavor $q$ ) follows a trajectory in 2-dimensional space. For the case of a massless quark flavor $q$ that trajectory is shown as the solid curve in Fig. 7, where we have chosen the values $\left(Q_{1}^{2}, Q_{2}^{2}, Q_{3}^{2}\right)=(4,10,80) \mathrm{GeV}^{2}$ for this example. The white (unshaded) area is the closed convex hull of the vectors that form the trajectory. Similar but slightly different trajectories are obtained for massive quark flavors, which are not shown here in order to keep the figure simple. As a consequence of the dipole picture the 2-vectors $\left(F_{2}\left(W, Q_{1}^{2}\right) / F_{2}\left(W, Q_{3}^{2}\right), F_{2}\left(W, Q_{2}^{2}\right) / F_{2}\left(W, Q_{3}^{2}\right)\right)^{\mathrm{T}}$ must lie within the closed convex hull of those trajectories, independently of the energy $W$, see (4.3). The dashed lines in Fig. 7 represent the two bounds (4.4) and (4.5). Clearly, the correlated bound (4.3) is much stronger than those separate bounds on the ratios.

Next we want to compare the stronger bound (4.3) with experimental data. For this purpose we need data points of $F_{2}$ at three different $Q_{i}^{2}$ but at the same $W$. However, most of the available data are not published in $\left(W, Q^{2}\right)$-binning. We have found only few points which are suitable for a direct comparison with our bound, that is with the same $W$ and three different $Q_{i}^{2}$ that are not too close to each other. We will now present two of these examples. Further below we will then again use the ALLM97 fit for a more comprehensive analysis of the kinematical range in which the bound (4.3) is respected. For the comparison with actual HERA data we choose

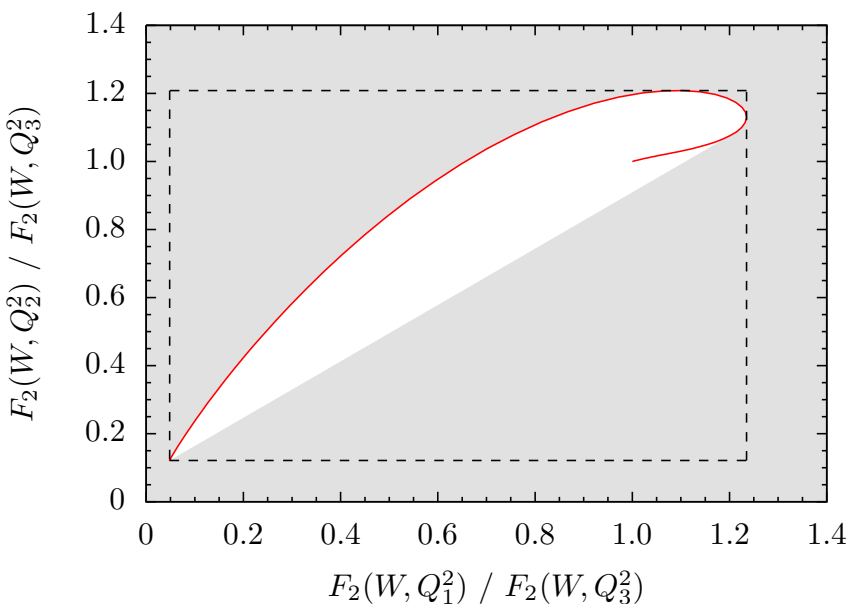

FIG. 7: The trajectory of the vectors $\left(f^{(q)}\left(r, Q_{1}^{2}\right) / f^{(q)}\left(r, Q_{3}^{2}\right), f^{(q)}\left(r, Q_{2}^{2}\right) / f^{(q)}\left(r, Q_{3}^{2}\right)\right)^{\mathrm{T}}$ for variation of $r$ (solid curve), here for a massless quark flavor $q$ and for the choice $\left(Q_{1}^{2}, Q_{2}^{2}, Q_{3}^{2}\right)=(4,10,80) \mathrm{GeV}^{2}$. The unshaded area is the closed convex hull of the vectors that form the trajectory. According to the weaker bounds (4.4) and (4.5) the vectors $\left(F_{2}\left(W, Q_{1}^{2}\right) / F_{2}\left(W, Q_{3}^{2}\right), F_{2}\left(W, Q_{2}^{2}\right) / F_{2}\left(W, Q_{3}^{2}\right)\right)^{T}$ must lie within the dashed rectangle, while the stronger bound (4.3) requires them to lie within the convex hull of the unshaded area and the corresponding areas obtained for massive quarks. The curves for massive quarks have a similar shape and are not shown here for simplicity.

as the observable the reduced cross section instead of $F_{2}$, since the former is the one which was directly measured. The reduced cross section is defined as

$$
\sigma_{\mathrm{r}}\left(W, Q^{2}\right)=\frac{Q^{2}}{4 \pi^{2} \alpha_{\mathrm{em}}}\left(\sigma_{T}+\frac{2(1-y)}{1+(1-y)^{2}} \sigma_{L}\right),
$$

with $y \approx\left(W^{2}+Q^{2}\right) / s$, see (2.2), where $\sqrt{s} \approx 300 \mathrm{GeV}$ is the lepton-proton center-of-mass energy for the available HERA data. It is straightforward to derive correlated bounds for ratios of reduced cross sections instead of $F_{2}$ structure functions from the dipole picture. The derivation is completely analogous to the one described above. We just have to replace $f^{(q)}$ by

$$
f_{\mathrm{r}}^{(q)}=Q^{2}\left[w_{T}^{(q)}\left(r, Q^{2}\right)+\frac{2(1-y)}{1+(1-y)^{2}} w_{L}^{(q)}\left(r, Q^{2}\right)\right],
$$

as can be seen from (2.14) and (4.6) together with (3.1), (3.2). The resulting bound is then as given by (4.3) but with $F_{2}$ replaced by $\sigma_{\mathrm{r}}$ and $f^{(q)}$ replaced by $f_{\mathrm{r}}^{(q)}$. Due to this modification the bound for the reduced cross section now depends on $W$ (which enters via $y$ ), which was not the case for the original bound for $F_{2}$. Fig. 8 confronts the bound on the quantity $\left(\sigma_{\mathrm{r}}\left(W, Q_{1}^{2}\right) / \sigma_{\mathrm{r}}\left(W, Q_{3}^{2}\right), \sigma_{\mathrm{r}}\left(W, Q_{2}^{2}\right) / \sigma_{\mathrm{r}}\left(W, Q_{3}^{2}\right)\right)^{\mathrm{T}}$ with its measured values from ZEUS [3] and H1 [2] for two different choices of $W$ and of the triple of $Q_{i}^{2}$. The depicted errors on the ratios are the combination of the experimental errors on $\sigma_{\mathrm{r}}$ in quadrature. The curves in 


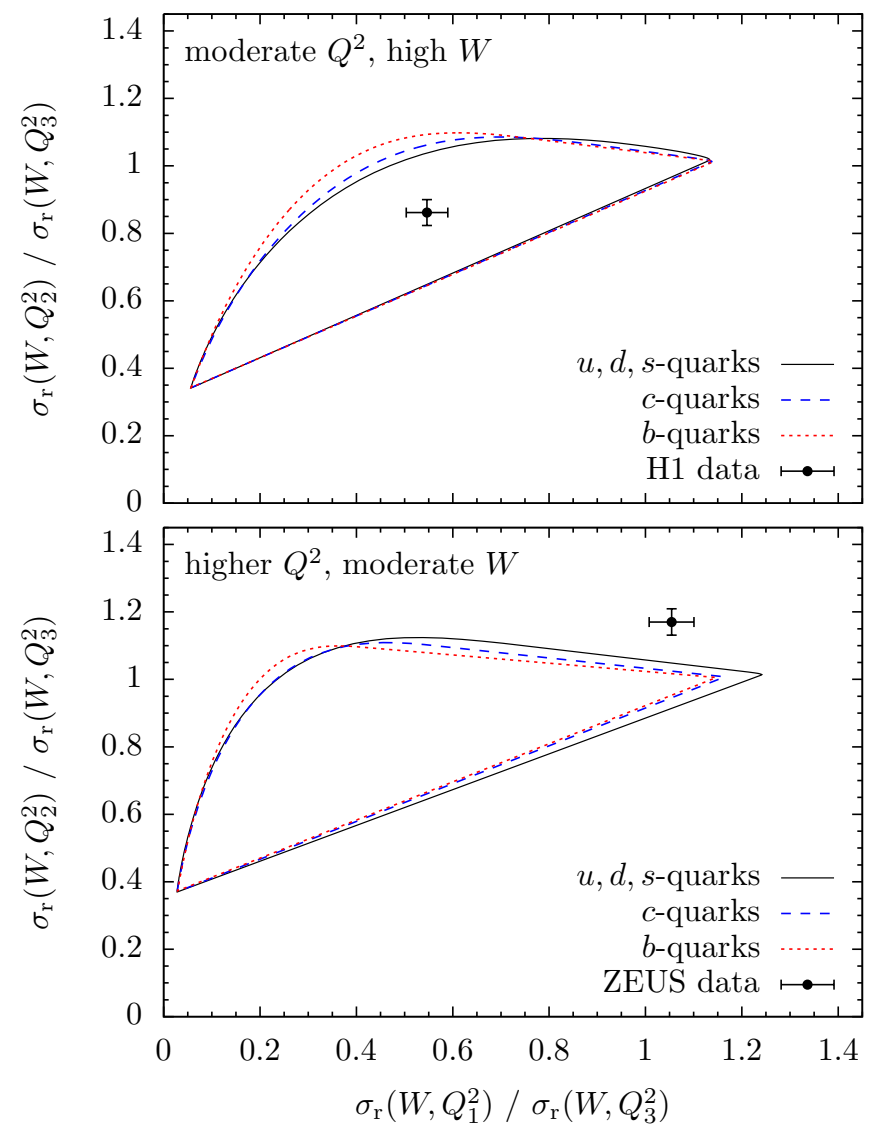

FIG. 8: Correlated bounds on ratios of the reduced cross section (4.6) at different values of $Q^{2}$ obtained from the dipole picture, confronted with HERA data. The inner parts of the different curves show the allowed regions if only contributions from specific quark flavors are considered, while the convex hull of these regions gives the net bound if no further assumptions are made. The kinematical values are $W=247 \mathrm{GeV},\left(Q_{1}^{2}, Q_{2}^{2}, Q_{3}^{2}\right)=(2,12,35) \mathrm{GeV}^{2}$ for the upper plot and $W=75 \mathrm{GeV},\left(Q_{1}^{2}, Q_{2}^{2}, Q_{3}^{2}\right)=(3.5,45,120) \mathrm{GeV}^{2}$ for the lower plot.

Fig. 8 show the correlated bounds for contributions to $\left(\sigma_{\mathrm{r}}\left(W, Q_{1}^{2}\right) / \sigma_{\mathrm{r}}\left(W, Q_{3}^{2}\right), \sigma_{\mathrm{r}}\left(W, Q_{2}^{2}\right) / \sigma_{\mathrm{r}}\left(W, Q_{3}^{2}\right)\right)^{\mathrm{T}}$ from different quark flavors as given by the analog of (4.3) for $\sigma_{\mathrm{r}}$. Only if the point obtained from the data lies within the convex hull of all these curves it can possibly be described in the framework of the dipole picture. We see that this condition is fulfilled for the high $W$, moderate $Q^{2}$ sample (upper graph), while it is violated by approximately two standard deviations for the lower $W$, higher $Q^{2}$ sample (lower graph).

The above discussion refers to the applicability of the dipole picture at a given value of $W$ for one particular triple of $Q^{2}$-values. For a determination of the range of applicability of the dipole picture it is more desirable to determine for a given $W$ a maximal range in $Q^{2}$ in which the three $Q_{i}^{2}$ can be chosen without giving rise to a violation of the bound. For this purpose we now consider again the structure function $F_{2}$ (and no longer the reduced cross section). Using the ALLM97 fit to the measured $F_{2}$ data we can then perform a continuous scan in $Q^{2}$ and determine precisely the kinematical range in which the bounds are respected. We first consider the correlated bounds obtained from (4.3), and later compare the allowed $Q^{2}$-range with the one resulting from the weaker bounds (4.4) and (4.5).

Let us first fix the energy $W$ at some value. We will in the following call a violation of the dipole-picture bound a 'significant' violation if the ALLM97 $F_{2}$ ratios give a relative deviation of more than $10 \%$ from the bound. This accounts for a kind of error band which should be associated with the ALLM97 fit or with the corresponding ratios of $F_{2}$. If for a certain triple $\left(Q_{1}^{2}, Q_{2}^{2}, Q_{3}^{2}\right)$ the ratios obtained from the ALLM97 fit violate the bounds by a significant amount (in the above sense) any $Q^{2}$-range containing the values $Q_{1}^{2}, Q_{2}^{2}, Q_{3}^{2}$ is excluded for a successful description within the dipole picture. In contrast, agreement with the bounds for a triple does not necessarily imply agreement for the full range $\left[\min _{i}\left(Q_{i}^{2}\right), \max _{i}\left(Q_{i}^{2}\right)\right]$ of that triple since the bounds depend on all three $Q_{i}^{2}$. We therefore systematically search for the maximal $Q^{2}$ range that contains no $Q^{2}$-triple for which the bounds are violated significantly. Technically, we do this by searching for the minimal $Q^{2}$-range in which we can find at least one $Q^{2}$-triple for which the bounds are violated significantly. The lower bound of a given $Q^{2}$-interval turns out to have only mild influence on whether a significant violation of the bounds can be found within that interval - provided it is not much larger than $1 \mathrm{GeV}^{2}$. We therefore keep the lower end of the considered $Q^{2}$-range fixed at $1 \mathrm{GeV}^{2}$ and determine the upper end $Q_{\max }^{2}$ of the $Q^{2}$-range within which the bounds are not significantly violated. We can then repeat this procedure for each energy $W$ and determine $Q_{\max }^{2}$ as a function of $W$.

The solid line in Fig. 9 shows the result of such a calculation based on the correlated bounds obtained from (4.3). The allowed $Q^{2}$-range slowly grows with increasing energy, as can be expected on general grounds. $Q_{\max }^{2}$ ranges from about $100 \mathrm{GeV}^{2}$ for $W=60 \mathrm{GeV}$ to about $200 \mathrm{GeV}^{2}$ for $W=245 \mathrm{GeV}$. The dashed line in Fig. 9 represents the analogous curve obtained from the uncorrelated bounds (4.4) and (4.5). Here we have varied both $Q_{1}^{2}$ and $Q_{3}^{2}$ in (4.4) in order to determine the maximal virtuality, $Q_{\max }^{2}$, below which both $Q_{1}^{2}$ and $Q_{3}^{2}$ can be chosen arbitrarily without giving rise to a significant violation of the bound. We see that the correlated bounds resulting from (4.3) indeed give stronger restrictions on the range of validity of the dipole picture than the uncorrelated bounds (4.4), (4.5).

Note that the violation of the correlated bound does not take place at a constant value of $x$. In the contrary, the value of $x$ changes along the solid line in Fig. 9. For $Q^{2}=100 \mathrm{GeV}^{2}$ we find that $x<0.03$ is required for the bound not to be violated, while for $Q^{2}=200 \mathrm{GeV}^{2}$ the bound is only respected for $x<0.003$. A similar observation applies to the uncorrelated bounds (4.4), (4.5) (see the dashed line in Fig. 9) as already observed in 24]. 


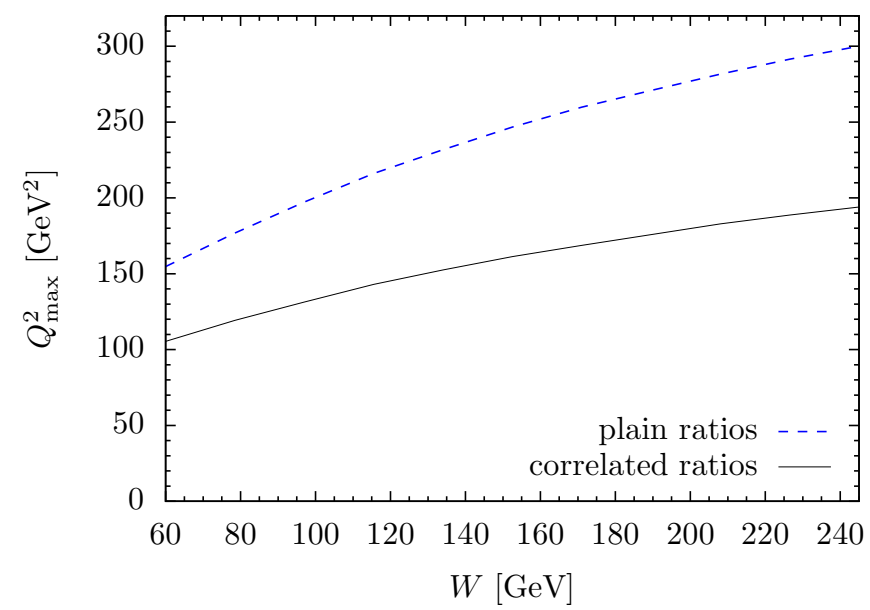

FIG. 9: Upper limit $Q_{\max }^{2}$ of the $Q^{2}$-range in which the ALLM97 fit to $F_{2}$ is consistent with the bounds obtained from the dipole picture within a $10 \%$ relative deviation of the $F_{2}$ ratios, plotted as a function of the energy $W$. The curves are for consistency with the correlated bounds (4.3) (solid line), and with the uncorrelated bounds (4.4) and (4.5) (dashed line), respectively.

Obviously, a violation of the above bounds indicates that some contributions to the cross section become important which are not contained in the dipole picture. We would like to emphasize that such corrections to the dipole picture might become sizable already before the bounds are actually violated. One should therefore expect that corrections to the standard dipole picture are important already if the data come close to the bounds.

The upper limit on the kinematical range of validity of the dipole picture that we find here appears to be rather low in view of the fact that phenomenological fits to $F_{2}$ data based on the dipole picture often work quite well up to rather high $Q^{2}$, see for example [8]. However, the good quality of those fits at large $Q^{2}$ is not in contradiction with our result. We recall that the bounds derived in this section crucially depend on the correct functional dependence of the dipole cross section $\hat{\sigma}$ on $r$ and $W$, as obtained naturally from the derivation of the dipole picture presented in [22, 23]. In particular, $\hat{\sigma}$ needs to be independent of $Q^{2}$ for our bounds to be valid. But almost all recent models for $\hat{\sigma}$ assume it to depend on $x$, and hence on $Q^{2}$. The transition from the energy variable $W$ to the energy variable $x$ in the dipole cross section requires additional assumptions the justification and the physical significance of which appears difficult to assess. In practice, they might capture - at least partly - some corrections that are left out in the usual dipole picture (see the discussion in section $\amalg$ ). It would be very desirable to obtain a better understanding of this situation. An important step would be to check whether it is also possible to describe the presently available HERA data by models for the dipole cross section based on the more natural variables $r$ and $W$.

We finally note that our bounds are modified if one uses, as suggested by Hand's convention, the relation (2.13) between $F_{2}$ and the $\gamma^{*} p$ cross sections $\sigma_{T}$ and $\sigma_{L}$ instead of the simpler relation (2.14) that has been used here. The modification becomes relevant for not so small $x$, and hence for large $Q^{2}$. The natural kinematical region for the application of the dipole picture is the region of small $x$, so that this modification is only of minor relevance for the dipole picture. Nevertheless, we find it interesting to see the effect of using (2.13) on our bounds and illustrate it in an example in appendix A. Our conclusions about the range of validity of the dipole picture would not to be significantly affected.

\section{CONCLUSIONS}

The dipole picture of high energy photon-proton scattering is a popular framework for the analysis and interpretation of HERA data. However, the dipole picture is not exact, and a number of assumptions and approximations are needed to obtain it from the general description of photon-proton scattering. It is therefore important to determine as precisely as possible its kinematical range of validity. Using the dipole picture beyond that range could clearly result in misleading conclusions.

In the present paper we have briefly summarized the assumptions and approximations underlying the dipole picture in addition to taking the high energy limit. In particular we have indicated some contributions to the $\gamma^{*} p$ cross section which are not contained in the dipole picture and might give rise to significant correction terms in some kinematical regions. We have then derived various bounds on ratios of deep inelastic structure functions from the dipole picture. These bounds involve only the photon wave functions and do not make use of any model assumptions about the dipole-proton cross section. They have to be respected in the kinematical range of applicability of the dipole picture. A comparison with experimental data then allowed us to constrain this range independently of any model assumptions.

We have first considered the structure functions $F_{L}$, $F_{2}^{(c)}$, and $F_{2}$, all taken at the same $W$ and $Q^{2}$. From the dipole picture we have obtained an upper bound on $F_{L} / F_{2}$ as well as a correlated upper bound on the ratios $F_{L} / F_{2}$ and $F_{2}^{(c)} / F_{2}$. It will be interesting to compare these bounds with future results from measurements of these structure functions.

Furthermore, we have derived correlated bounds on ratios of $F_{2}$ at three different $Q_{i}^{2}$ but at the same energy $W$. These bounds are significantly more restrictive than bounds on simple ratios that had been obtained already in [24]. We have compared these bounds with experimental data. More precisely, we have used the ALLM97 fit to the measured $F_{2}$ data except for two examples in which we have used actual data points. Since our bounds apply to ratios of $F_{2}$ at the same $W$ a more direct comparison would require to have the data in $\left(W, Q^{2}\right)$-binning instead of the commonly used $\left(x, Q^{2}\right)$ binning. Employing 
the ALLM97 fit within the kinematical range of HERA we have computed ranges in $Q^{2}$ in which the bounds obtained from the dipole picture are respected. We have further studied the dependence of these ranges on the energy $W$. Depending on $W$ the dipole picture fails to be applicable above a $Q^{2}$ of about 100 to $200 \mathrm{GeV}^{2}$. We expect that already for values of $Q^{2}$ somewhat below those limits corrections to the usual dipole picture become important. For low $Q^{2}$ the bounds on ratios of $F_{2}$ are found to be respected by the data.

We should point out that the bounds on ratios of $F_{L}$, $F_{2}^{(c)}$ and $F_{2}$ obtained in section $\amalg$ are independent of the choice of energy variable in the dipole cross section $\hat{\sigma}$. The bounds on ratios of $F_{2}$ at the same $W$ but different $Q_{i}^{2}$ discussed in section [V] on the other hand, crucially require that $\hat{\sigma}$ is independent of $Q^{2}$. Similarly, other modifications of the standard dipole picture formulae (2.18)-(2.21) might in general affect the bounds resulting from these formulae. For an example see [29].

We recall that in 23, 24] an upper bound on the ratio $R=\sigma_{L} / \sigma_{T}$ has been derived from the dipole picture, $R \leq 0.372$. The experimental data for that ratio have large errors but appear to come close to the bound for $Q^{2}$ below about $2 \mathrm{GeV}^{2}$, which can be interpreted as a possible breakdown of the dipole picture in this region of low $Q^{2}$. Combining this with the findings of the present paper we conclude that the data are compatible with the bounds resulting from the dipole picture for $Q^{2}$ between $2 \mathrm{GeV}^{2}$ and $100-200 \mathrm{GeV}^{2}$, with the upper limit depending on $W$. Any results that depend on using the dipole picture outside this kinematical range might be considerably affected by potential corrections to the standard dipole picture and should be interpreted only with great care.

\section{Acknowledgments}

We thank M. Diehl, J. Forshaw, H. Kowalski, M. Ryskin, D. Schildknecht, and G. Shaw for useful discussions. This work was supported by the Deutsche Forschungsgemeinschaft, project number NA 296/4-1.

\section{APPENDIX A: THE NORMALIZATION OF $\gamma^{*} p$ CROSS SECTIONS AND ITS CONSEQUENCES IN THE DIPOLE PICTURE}

In this appendix we would like to illustrate in one example how our bounds are affected by different normalizations of the $\gamma^{*} p$ cross sections $\sigma_{T}$ and $\sigma_{L}$ relative to $F_{2}$. In the context of high energy scattering, in particular when using the dipole picture, one usually employs the simple relation (2.14). The relation (2.13) derived from Hand's convention reduces to that simple expression if the high energy limit is taken for fixed $Q^{2}$. For finite $x$ the normalization of $\sigma_{T, L}$ relative to $F_{2}$ differs by a factor $(1-x)$ between the two choices (neglecting terms of

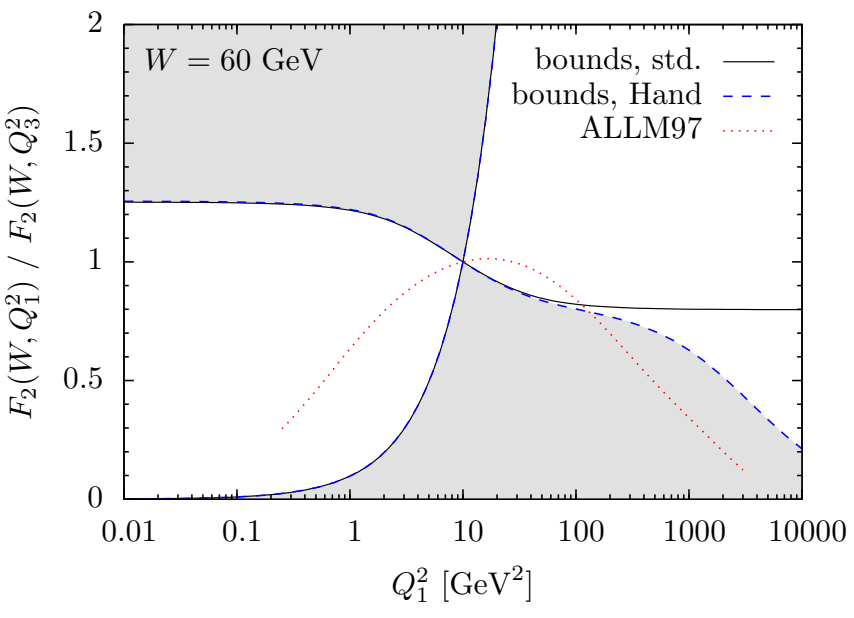

FIG. 10: Change of the bound (4.4) on $F_{2}\left(W, Q_{1}^{2}\right) / F_{2}\left(W, Q_{3}^{2}\right)$ due to using Hand's convention (2.13) instead of the simpler (2.14), shown here for $W=60 \mathrm{GeV}$ and the choice $Q_{3}^{2}=$ $10 \mathrm{GeV}^{2}$. The solid lines are the original bounds (4.4), while the dashed lines represent the modified bounds. The dotted line is the ALLM97 fit to $F_{2}\left(W, Q_{1}^{2}\right) / F_{2}\left(W, Q_{3}^{2}\right)$.

$\left.\mathcal{O}\left(m_{p}^{2} / W^{2}\right)\right)$, as is indicated in (2.13).

The bounds discussed in the present paper are all based on the simpler formula (2.14). It is straightforward, however, to derive similar bounds based on the relation (2.13). The additional factor $(1-x)$ depends both on $Q^{2}$ and $W$. Therefore this factor does not cancel if ratios of structure functions are taken at different $Q_{i}^{2}$. Furthermore, the bounds on ratios of $F_{2}$ inherit a dependence on $W$ from this factor. The bounds on ratios of $F_{L}, F_{2}^{(c)}$, and $F_{2}$ discussed in section [II], on the other hand, are not affected. There the structure functions are evaluated at the same $W$ and $Q^{2}$ and the additional factor $(1-x)$ cancels in the ratios.

In Fig. 10 we show for one energy $W=60 \mathrm{GeV}$ how the bound (4.4) is changed if one uses (2.13) instead of (2.14). A sizable deviation from the original bound occurs only at relatively large $Q^{2}$ where the new bound is closer to the data than the original bound. However, both bounds are violated by the data at about the same $Q^{2}$ and the difference between the original and the modified bound grows only at larger $Q^{2}$. Similar remarks apply to the correlated bounds on ratios of $F_{2}$. Therefore the normalization of the $\gamma^{*} p$ cross sections according to Hand's convention would not significantly alter our results concerning the range of validity of the dipole picture.

\section{APPENDIX B: CONVEX HULLS, CONVEX CONES AND MOMENT PROBLEMS}

In this appendix we discuss the notions of convex hull and convex cone as well as some further mathematical relations. The precise mathematical definitions can be found in [30], for our notation see also [31]. 
Let us consider the $n$-dimensional Euclidean space $\mathbb{R}^{n}$ with elements $\boldsymbol{x}, \boldsymbol{y}$ etc. A non-empty subset $X$ of $\mathbb{R}^{n}$ is called a convex set if for any elements $\boldsymbol{x}, \boldsymbol{y}$ in $X$ and any real number $a$ with $0 \leq a \leq 1$ the element $a \boldsymbol{x}+(1-a) \boldsymbol{y}$ is also contained in $X$. That is, with any two points of $X$ the complete straight line connecting them is also in $X$.

Let now $Y$ be an arbitrary nonempty subset of $\mathbb{R}^{n}$. The minimum convex set containing $Y$ exists [30] and is called the convex hull of $Y$ and denoted by $\operatorname{co}(Y)$. Its closure is denoted by $\overline{\mathrm{co}}(Y)$. To illustrate this concept we give a physical example. Let $Y=\left\{\boldsymbol{y}^{(1)}, \ldots, \boldsymbol{y}^{(N)}\right\}$ be a set of $N$ points in $\mathbb{R}^{n}$. Consider arbitrary distributions of masses $m_{i} \geq 0(i=1, \ldots, N)$ on these points. The center of mass is then

$$
\boldsymbol{x}=\frac{\sum_{i=1}^{N} m_{i} \boldsymbol{y}^{(i)}}{\sum_{i=1}^{N} m_{i}}
$$

The convex hull of $Y, \operatorname{co}(Y)$, is the set of all possible center of mass points of such mass distributions.

Next we discuss the notion of convex cone. A nonempty subset $X$ of $\mathbb{R}^{n}$ is called a convex cone if for any elements $\boldsymbol{x}, \boldsymbol{y}$ of $X$ and any real number $a \geq 0$ the elements $a \boldsymbol{x}$ and $\boldsymbol{x}+\boldsymbol{y}$ are also contained in $X$. Let $Y$ be an arbitrary non-empty subset of $\mathbb{R}^{n}$, then the minimal convex cone containing $Y$ exists and is denoted by $\mathrm{K}(Y)$. Its closure is denoted by $\overline{\mathrm{K}}(Y)$.

We illustrate these notions with a two-dimensional example. Let $Y$ consist of three points in $\mathbb{R}^{2}$

$$
Y=\left\{\boldsymbol{y}^{(1)}, \boldsymbol{y}^{(2)}, \boldsymbol{y}^{(3)}\right\}
$$

as shown in Fig. 11. Here

$$
\boldsymbol{y}^{(i)}=\left(\begin{array}{c}
y_{1}^{(i)} \\
y_{2}^{(i)}
\end{array}\right)
$$

and we suppose

$$
y_{2}^{(i)}>0 \quad \text { for } i=1,2,3 .
$$

The convex hull of $Y, \operatorname{co}(Y)$, is given by the dark grey triangle bounded by the polygon from $\boldsymbol{y}^{(1)}$ to $\boldsymbol{y}^{(2)}, \boldsymbol{y}^{(3)}$ and back to $\boldsymbol{y}^{(1)}$. The cone $\mathrm{K}(Y)$ is indicated by the light grey area bounded by the rays $\lambda \boldsymbol{y}^{(2)}$ with $\lambda \geq 0$ and $\mu \boldsymbol{y}^{(3)}$ with $\mu \geq 0$.

Let $Y$ be a non-empty subset of $\mathbb{R}^{n}$ and let co( $\left.Y\right)$ be the convex hull of $Y$ and $\mathrm{K}(Y)$ the minimal convex cone containing $Y$. We define the set

$$
\mathrm{K}^{\prime}(Y)=\{\boldsymbol{x} \mid \boldsymbol{x}=\lambda \boldsymbol{y}, \lambda \geq 0, \boldsymbol{y} \in \operatorname{co}(Y)\}
$$

and assert that

$$
\mathrm{K}^{\prime}(Y)=\mathrm{K}(Y) .
$$

The proof of (B6) goes as follows. It is easy to see that $\mathrm{K}^{\prime}(Y)$ is a convex cone containing $Y$. Thus, since $\mathrm{K}(Y)$ is the minimal such cone we have

$$
\mathrm{K}^{\prime}(Y) \supset \mathrm{K}(Y) .
$$

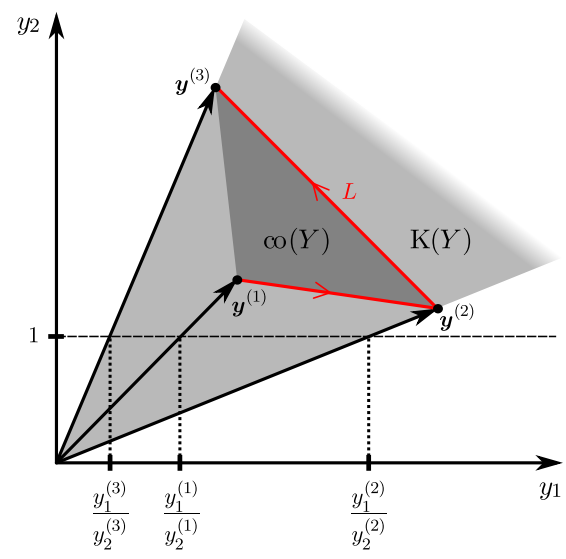

FIG. 11: Illustration of the convex hull $\operatorname{co}(Y)$ and the convex cone $\mathrm{K}(Y)$ for the set $Y$ of (B2). Here both sets are closed, that is $\overline{\mathrm{co}}(Y)=\operatorname{co}(Y)$ and $\overline{\mathrm{K}}(Y)=\mathrm{K}(Y)$.

On the other hand, $\mathrm{K}(Y)$ is a convex set containing $Y$ and $\operatorname{co}(Y)$ is the minimal such set. Thus

$$
\mathrm{K}(Y) \supset \operatorname{co}(Y) .
$$

Since $\mathrm{K}(Y)$ is a convex cone this implies that for any element $\boldsymbol{x} \in \operatorname{co}(Y)$ and any $\lambda \geq 0$ we have $\lambda \boldsymbol{x} \in \mathrm{K}(Y)$. That is, we have

$$
\mathrm{K}^{\prime}(Y) \subset \mathrm{K}(Y) .
$$

Therefore, we have shown that $\mathrm{K}^{\prime}(Y)=\mathrm{K}(Y)$. That is, every element of $\mathrm{K}(Y)$ can be written in the form given in (B5). For the closures we find in a similar way

$$
\overline{\mathrm{K}}(Y)=\{\boldsymbol{x} \mid \boldsymbol{x}=\lambda \boldsymbol{y}, \lambda \geq 0, \boldsymbol{y} \in \overline{\mathrm{co}}(Y)\} .
$$

Next we come to the moment problem which is at the heart of our derivations of bounds. Suppose we have a continuous vector function on a closed interval $\left[t_{0}, t_{1}\right] \subset$ $\mathbb{R}$ defining a curve $L$ in $\mathbb{R}^{n}$ :

$$
\begin{aligned}
L: \quad\left[t_{0}, t_{1}\right] & \rightarrow \mathbb{R}^{n}, \\
t & \mapsto \boldsymbol{y}(t) .
\end{aligned}
$$

We also suppose that there is at least one constant vector $\boldsymbol{a}$ such that

$$
\boldsymbol{a}^{\mathrm{T}} \boldsymbol{y}(t)>0 \quad \text { for all } t \in\left[t_{0}, t_{1}\right] .
$$

We are interested in the set $\tilde{K}$ of all points $\boldsymbol{x}$ of $\mathbb{R}^{n}$ which can be represented as

$$
\boldsymbol{x}=\int_{t_{0}}^{t_{1}} \boldsymbol{y}(t) \mathrm{d} \Sigma(t)
$$

where $\Sigma(t)$ is some non-decreasing function on $\left[t_{0}, t_{1}\right]$. Note that such a function is bounded from below and above since

$$
\Sigma\left(t_{0}\right) \leq \Sigma(t) \leq \Sigma\left(t_{1}\right) .
$$


Before we discuss the solution of this problem as given in 32] we note that in (B13) we are dealing with so-called Stieltjes integrals, see for example [33, 34]. The reader not familiar with these integrals may always set

$$
\mathrm{d} \Sigma(t)=\sigma(t) \mathrm{d} t
$$

where $\sigma(t)$ is some non-negative distribution. That is, $\sigma(t)$ can be an ordinary non-negative function but can also contain non-negative $\delta$-distributions.

The solution of the problem posed above is as follows, see [32]. The set $\tilde{K}$ of points which can be represented in the form (B13) is given by $\overline{\mathrm{K}}(L)$, that is, by the smallest closed convex cone containing the curve $L$ :

$$
\tilde{K}=\overline{\mathrm{K}}(L) .
$$

Consider for illustration the two-dimensional example as in Fig. 11 and the following curve $L$ defined for $t \in$ $[0,1]$,

$L: t \mapsto \boldsymbol{y}(t)= \begin{cases}\boldsymbol{y}^{(1)}+2 t\left(\boldsymbol{y}^{(2)}-\boldsymbol{y}^{(1)}\right) & : 0 \leq t \leq \frac{1}{2}, \\ \boldsymbol{y}^{(2)}+(2 t-1)\left(\boldsymbol{y}^{(3)}-\boldsymbol{y}^{(2)}\right): \frac{1}{2}<t \leq 1\end{cases}$

We are interested in the points $\boldsymbol{x}$ allowing a representation

$$
\boldsymbol{x}=\int_{0}^{1} \boldsymbol{y}(t) \mathrm{d} \Sigma(t)
$$

with some non-decreasing function $\Sigma(t)$. According to the theorem quoted above $\boldsymbol{x}$ has to be in the closed convex cone $\overline{\mathrm{K}}(L)$ as shown in Fig. 11. We ask now for the allowed range for $x_{1}$ given some $x_{2}$. The possible $x_{1}$ values are obtained by cutting the cone $\overline{\mathrm{K}}(L)$ at $y_{2}=x_{2}=$ const. and reading off the corresponding $y_{1}$ values. Similarly, the allowed range of the ratio $x_{1} / x_{2}$ is obtained if we choose $y_{2}=1$ for cutting the cone. Clearly, to get the extremal values of $x_{1} / x_{2}$ we just have to consider the generating rays $\lambda \boldsymbol{y}^{(i)}, \lambda \geq 0$, for $i=1,2,3$. Cutting them at $y_{2}=1$ gives $y_{1}^{(i)} / y_{2}^{(i)}, i=1,2,3$. Among these ratios there are the extremal points of $x_{1} / x_{2}$. In our example we get

$$
\frac{y_{1}^{(3)}}{y_{2}^{(3)}} \leq \frac{x_{1}}{x_{2}} \leq \frac{y_{1}^{(2)}}{y_{2}^{(2)}} .
$$

Note that the interval $\left[y_{1}^{(3)} / y_{2}^{(3)}, y_{1}^{(2)} / y_{2}^{(2)}\right]$ is the convex hull of the set $\left\{y_{1}^{(i)} / y_{2}^{(i)} \mid i=1,2,3\right\}$.

For the general case, in $\mathbb{R}^{n}$, the situation is completely analogous. Consider (B13) in $\mathbb{R}^{n}(n \geq 2)$ and let us write in components

$$
\left(\begin{array}{c}
x_{1} \\
\vdots \\
x_{n}
\end{array}\right)=\int_{t_{0}}^{t_{1}}\left(\begin{array}{c}
y_{1}(t) \\
\vdots \\
y_{n}(t)
\end{array}\right) \mathrm{d} \Sigma(t)
$$

Let us suppose that

$$
0<c_{0} \leq y_{n}(t) \leq c_{1} \quad \text { for } t_{0} \leq t \leq t_{1} .
$$

Thereby (B12) is satisfied with $\boldsymbol{a}^{\mathrm{T}}=(0, \ldots, 0,1)$. To get the bounds for the ratio vector

$$
\boldsymbol{x}^{\prime}=\left(\begin{array}{c}
x_{1} / x_{n} \\
\vdots \\
x_{n-1} / x_{n} \\
1
\end{array}\right)
$$

we just have to cut the cone $\overline{\mathrm{K}}(L)$ with the hyperplane $x_{n}=1$. The corresponding set in $\mathbb{R}^{n}$ obtained by this cutting is given by the closed convex hull of the ratio vectors of the curve $L$ generating the cone $\overline{\mathrm{K}}(L)$. That is, we denote by $L^{\prime}$ the following curve in $\mathbb{R}^{n}$

$$
L^{\prime}: \quad t \mapsto\left(\begin{array}{c}
y_{1}(t) / y_{n}(t) \\
\vdots \\
y_{n-1}(t) / y_{n}(t) \\
1
\end{array}\right), \quad t \in\left[t_{0}, t_{1}\right] .
$$

Let $\overline{\mathrm{co}}\left(L^{\prime}\right)$ be the closed convex hull of $L^{\prime}$. The intersetion of $\overline{\mathrm{K}}(L)$ with the hyperplane $x_{n}=1$ is given by $\overline{\mathrm{co}}\left(L^{\prime}\right)$. Clearly, the extremal points of the above conehyperplane intersection must be given by the intersections of the rays generating the cone $\overline{\mathrm{K}}(L)$, that is by the rays through the curve $L$. But this gives just $L^{\prime}$.

We give now a formal proof of the above statements. For this consider the minimal closed convex cone $\overline{\mathrm{K}}(L)$ containing $L$ and analogously $\overline{\mathrm{K}}\left(L^{\prime}\right)$ containing $L^{\prime}$. We assert that

$$
\overline{\mathrm{K}}(L)=\overline{\mathrm{K}}\left(L^{\prime}\right)
$$

To prove (B24) we note that according to (B13) and (B23) all vectors $\boldsymbol{x}^{\prime} \in \overline{\mathrm{K}}\left(L^{\prime}\right)$ are of the form

$$
\boldsymbol{x}^{\prime}=\int_{t_{0}}^{t_{1}} \frac{\boldsymbol{y}(t)}{y_{n}(t)} \mathrm{d} \Sigma^{\prime}(t)
$$

with some non-decreasing function $\Sigma^{\prime}(t)$. Due to (B21) the division by $y_{n}(t)$ in (B25) is harmless and we can define a non-decreasing function $\Sigma(t)$ on $\left[t_{0}, t_{1}\right]$ by

$$
\Sigma(t)=\int_{t_{0}}^{t} \frac{1}{y_{n}\left(t^{\prime}\right)} \mathrm{d} \Sigma^{\prime}\left(t^{\prime}\right)
$$

We get then for $\boldsymbol{x}^{\prime}$ of (B25)

$$
\boldsymbol{x}^{\prime}=\int_{t_{0}}^{t_{1}} \boldsymbol{y}(t) \mathrm{d} \Sigma(t)
$$

That is, $\boldsymbol{x}^{\prime} \in \overline{\mathrm{K}}(L)$ according to (B13) and we have shown

$$
\overline{\mathrm{K}}\left(L^{\prime}\right) \subset \overline{\mathrm{K}}(L) \text {. }
$$

Now we consider an arbitrary element $\boldsymbol{x} \in \overline{\mathrm{K}}(L)$ which according to (B13) has the form

$$
\boldsymbol{x}=\int_{t_{0}}^{t_{1}} \boldsymbol{y}(t) \mathrm{d} \Sigma(t)
$$


with some non-decreasing function $\Sigma(t)$. We define a non-decreasing function $\Sigma^{\prime}(t)$ on $\left[t_{0}, t_{1}\right]$ by

$$
\Sigma^{\prime}(t)=\int_{t_{0}}^{t} y_{n}\left(t^{\prime}\right) \mathrm{d} \Sigma\left(t^{\prime}\right)
$$

Again, we use here (B21). We get then

$$
\boldsymbol{x}=\int_{t_{0}}^{t_{1}} \boldsymbol{y}(t) \frac{1}{y_{n}(t)} \mathrm{d} \Sigma^{\prime}(t) .
$$

That is, $\boldsymbol{x} \in \overline{\mathrm{K}}\left(L^{\prime}\right)$ and therefore

$$
\overline{\mathrm{K}}(L) \subset \overline{\mathrm{K}}\left(L^{\prime}\right) .
$$

From (B28) and (B32) follows (B24), q.e.d.

From (B31) we can now draw the following conclusion for any non-zero element $\boldsymbol{x} \in \overline{\mathrm{K}}(L)$. Such an $\boldsymbol{x}$ is of the form (B29) with $\Sigma(t) \neq$ const. We have then with $\Sigma^{\prime}(t)$ from (B30),

$$
x_{n}=\Sigma^{\prime}\left(t_{1}\right)=\int_{t_{0}}^{t_{1}} \mathrm{~d} \Sigma^{\prime}(t)>0
$$

where we use (B21). From (B31) we can represent $\boldsymbol{x}$ as

$$
\boldsymbol{x}=x_{n} \boldsymbol{x}^{\prime}
$$

where

$$
\begin{array}{r}
\boldsymbol{x}^{\prime}=\int_{t_{0}}^{t_{1}}\left(\begin{array}{c}
y_{1}(t) / y_{n}(t) \\
\vdots \\
y_{n-1}(t) / y_{n}(t) \\
1
\end{array}\right) \mathrm{d} \Sigma^{\prime \prime}(t), \\
\mathrm{d} \Sigma^{\prime \prime}(t)=\frac{1}{x_{n}} \mathrm{~d} \Sigma^{\prime}(t), \quad \int_{t_{0}}^{t_{1}} \mathrm{~d} \Sigma^{\prime \prime}(t)=1 .
\end{array}
$$

Clearly $\boldsymbol{x}^{\prime}$ is in the intersection of $\overline{\mathrm{K}}\left(L^{\prime}\right)$ with the hyperplane $x_{n}^{\prime}=1$. Since $\overline{\mathrm{K}}\left(L^{\prime}\right)$ is the minimal closed convex cone containing $L^{\prime}$ this intersection is the minimal closed convex set containing $L^{\prime}$, that is, the closed convex hull $\overline{\mathrm{CO}}\left(L^{\prime}\right)$ :

$$
\boldsymbol{x}^{\prime} \in \overline{\mathrm{cO}}\left(L^{\prime}\right) .
$$

Thus we have shown that every non-zero vector $\boldsymbol{x} \in$ $\overline{\mathrm{K}}(L)$, that is of the form (B29), can be represented as $x_{n} \boldsymbol{x}^{\prime}$ where $\boldsymbol{x}^{\prime} \in \overline{\mathrm{co}}\left(L^{\prime}\right)$.

Now we come to the application of the above mathematical theorems to our problems. Consider three structure functions as in (4.1) but - for simplicity - only for fixed massless flavor $q$

$$
\left(\begin{array}{l}
F_{2}^{(q)}\left(W, Q_{1}^{2}\right) \\
F_{2}^{(q)}\left(W, Q_{2}^{2}\right) \\
F_{2}^{(q)}\left(W, Q_{3}^{2}\right)
\end{array}\right)=\int_{0}^{\infty} \mathrm{d} r r \frac{\hat{\sigma}^{(q)}(r, W)}{2 \pi \alpha_{\mathrm{em}}}\left(\begin{array}{l}
f^{(q)}\left(r, Q_{1}^{2}\right) \\
f^{(q)}\left(r, Q_{2}^{2}\right) \\
f^{(q)}\left(r, Q_{3}^{2}\right)
\end{array}\right) .
$$

To bring this into the form of the problem (B11), (B13), we change variables and set

$$
r=r_{0} \frac{t}{1-t}, \quad r_{0}=1 \mathrm{fm}, \quad 0<t<1 .
$$

Furthermore, we shall split off from $f^{(q)}\left(r, Q^{2}\right)$ the asymptotic terms for $r \rightarrow \infty$ and $r \rightarrow 0$. In the present case of a massless flavor $q, f^{(q)}\left(r, Q^{2}\right)$ decreases for $r \rightarrow \infty$ as $1 / r^{4}$, for $r \rightarrow 0$ it behaves as $1 / r^{2}$. Thus, we define a function

$$
g(r)=\frac{r_{0}^{4}}{r^{2}\left(r_{0}+r\right)^{2}}
$$

which is independent of $Q^{2}$. This allows us to define the function

$$
\hat{f}^{(q)}\left(t, Q^{2}\right)=\left\{\begin{aligned}
\left.\lim _{t^{\prime} \rightarrow 0} \frac{f^{(q)}\left(r, Q^{2}\right)}{g(r)}\right|_{r=r_{0} \frac{t^{\prime}}{1-t^{\prime}}} & \text { if } t=0, \\
\left.\frac{f^{(q)}\left(r, Q^{2}\right)}{g(r)}\right|_{r=r_{0} \frac{t}{1-t}} & \text { if } 0<t<1, \\
\left.\lim _{t^{\prime} \rightarrow 1} \frac{f^{(q)}\left(r, Q^{2}\right)}{g(r)}\right|_{r=r_{0} \frac{t^{\prime}}{1-t^{\prime}}} & \text { if } t=1
\end{aligned}\right.
$$

for all $t$ in the closed interval $[0,1]$, since the limites in (B40) exist. It is easy to show that $\hat{f}^{(q)}\left(t, Q^{2}\right)$ is continuous as function of $t$. Moreover, we find

$$
0<c_{0}\left(Q^{2}\right) \leq \hat{f}^{(q)}\left(t, Q^{2}\right) \leq c_{1}\left(Q^{2}\right)
$$

for all $t \in[0,1]$. Here $c_{j}\left(Q^{2}\right)(j=0,1)$ are fixed positive constants for fixed $Q^{2}$. Next we note that the dipole model makes only sense if the dipole cross section $\hat{\sigma}^{(q)}(r, W)$ can be integrated with $g(r)$, that is, if

$$
\int_{0}^{\infty} \mathrm{d} r r g(r) \frac{\hat{\sigma}^{(q)}(r, W)}{2 \pi \alpha_{\mathrm{em}}}<\infty .
$$

Further, we assume for $0<r<\infty$ :

$$
\hat{\sigma}^{(q)}(r, W) \geq 0 .
$$

This allows us to define a function $\Sigma^{(q)}(t, W)$ which is non-decreasing in $t$ for fixed $W$ :

$$
\Sigma^{(q)}(t, W)=\int_{0}^{t} \mathrm{~d} t^{\prime}\left[\frac{\mathrm{d} r^{\prime}}{\mathrm{d} t^{\prime}} r^{\prime} g\left(r^{\prime}\right) \frac{\hat{\sigma}^{(q)}\left(r^{\prime}, W\right)}{2 \pi \alpha_{\mathrm{em}}}\right]_{r^{\prime}=r_{0} \frac{t^{\prime}}{1-t^{\prime}}}
$$

for $0 \leq t \leq 1$. Conversely, every non-decreasing function $\Sigma^{(q)}(t, W)$ gives, via (B44), an acceptable dipole cross section $\hat{\sigma}^{(q)}(r, W)$. Furthermore, we define the curves $L$ and $L^{\prime}$ as follows

$$
\begin{aligned}
L: \quad t \mapsto \boldsymbol{y}(t):=\left(\begin{array}{c}
\hat{f}^{(q)}\left(t, Q_{1}^{2}\right) \\
\hat{f}^{(q)}\left(t, Q_{2}^{2}\right) \\
\hat{f}^{(q)}\left(t, Q_{3}^{2}\right)
\end{array}\right), \quad 0 \leq t \leq 1, \\
L^{\prime}: \quad t \mapsto \boldsymbol{y}^{\prime}(t):=\left(\begin{array}{c}
y_{1}(t) / y_{3}(t) \\
y_{2}(t) / y_{3}(t) \\
1
\end{array}\right), \quad 0 \leq t \leq 1 .
\end{aligned}
$$


Our original integrals (B37) take now exactly the form of (B13):

$$
\boldsymbol{x} \equiv\left(\begin{array}{l}
F_{2}^{(q)}\left(W, Q_{1}^{2}\right) \\
F_{2}^{(q)}\left(W, Q_{2}^{2}\right) \\
F_{2}^{(q)}\left(W, Q_{3}^{2}\right)
\end{array}\right)=\int_{0}^{1} \boldsymbol{y}(t) \mathrm{d} \Sigma^{(q)}(t, W)
$$

The vector function $\boldsymbol{y}(t)$ is continuous for $t \in[0,1]$ and (B12) is satisfied with $\boldsymbol{a}^{\mathrm{T}}=(0,0,1)$ due to (B41) which also guarantees (B21). From (B16) we conclude that $\boldsymbol{x}$ must be in the smallest closed convex cone containing $L$, that is, in $\overline{\mathrm{K}}(L)$. It is easy to see that this cone coincides with the cone defined as in (4.2) but for fixed flavor $q$. Indeed, we have from (B10) that $\overline{\mathrm{K}}(L)$ can be represented as

$$
\overline{\mathrm{K}}(L)=\{\boldsymbol{x} \mid \boldsymbol{x}=\lambda \boldsymbol{z}, \lambda \geq 0, \boldsymbol{z} \in \overline{\mathrm{co}}(L)\} .
$$

With a simple rescaling by $g(r)$ from (B39) and using that the closure takes care of the limiting points $t=0$ and $t=1$ which correspond to $r \rightarrow 0$ and $r \rightarrow \infty$ we get for every $\boldsymbol{x} \in \overline{\mathrm{K}}(L)$ the representation

$$
\begin{aligned}
& \boldsymbol{x}=\boldsymbol{\mu} \boldsymbol{v}, \\
& \text { with } \quad \mu \geq 0, \quad \boldsymbol{v} \in \overline{\mathrm{co}}\left\{\left(\begin{array}{l}
f^{(q)}\left(r, Q_{1}^{2}\right) \\
f^{(q)}\left(r, Q_{2}^{2}\right) \\
f^{(q)}\left(r, Q_{3}^{2}\right)
\end{array}\right) \mid r \in \mathbb{R}^{+}\right\} .
\end{aligned}
$$

The extension of these arguments to more than one flavor is straightforward. With this we have given a rigorous proof of (4.2).
Consider next the ratio vector

$$
\boldsymbol{x}^{\prime}=\frac{1}{x_{3}} \boldsymbol{x}=\left(\begin{array}{c}
F_{2}^{(q)}\left(W, Q_{1}^{2}\right) / F_{2}^{(q)}\left(W, Q_{3}^{2}\right) \\
F_{2}^{(q)}\left(W, Q_{2}^{2}\right) / F_{2}^{(q)}\left(W, Q_{3}^{2}\right) \\
1
\end{array}\right) .
$$

From (B29), (B34) and (B36) we see that $\boldsymbol{x}^{\prime}$ must be in the closed convex hull $\overline{\mathrm{co}}\left(L^{\prime}\right)$ :

$$
\boldsymbol{x}^{\prime} \in \overline{\mathrm{co}}\left(L^{\prime}\right)
$$

We have from (B46), (B45) and (B40)

$$
\begin{aligned}
\overline{\operatorname{co}}\left(L^{\prime}\right) & =\overline{\operatorname{co}}\left\{\left(\begin{array}{c}
\hat{f}^{(q)}\left(t, Q_{1}^{2}\right) / \hat{f}^{(q)}\left(t, Q_{3}^{2}\right) \\
\hat{f}^{(q)}\left(t, Q_{2}^{2}\right) / \hat{f}^{(q)}\left(t, Q_{3}^{2}\right) \\
1
\end{array}\right) \mid 0 \leq t \leq 1\right\} \\
& =\overline{\operatorname{co}}\left\{\left(\begin{array}{c}
f^{(q)}\left(r, Q_{1}^{2}\right) / f^{(q)}\left(r, Q_{3}^{2}\right) \\
f^{(q)}\left(r, Q_{2}^{2}\right) / f^{(q)}\left(r, Q_{3}^{2}\right) \\
1
\end{array}\right) \mid 0<r<\infty\right\} .
\end{aligned}
$$

Taking the closure eliminates differences which could otherwise exist between the two convex hulls of the sets in (B52) originating from the fact that $\hat{f}^{(q)}\left(t, Q^{2}\right)$ is defined on a closed $t$ interval whereas $f^{(q)}\left(r, Q^{2}\right)$ is defined on an open $r$ interval. The straightforward extension of (B51) and (B52) to the case of several flavors $q$ proves (4.3).

With this we have illustrated for one particular case how our bounds are derived in a mathematically rigorous way. For all other cases analogous arguments can be applied.
[1] J. Breitweg et al. [ZEUS Collaboration], Phys. Lett. B 487 (2000) 53 arXiv:hep-ex/0005018.

[2] C. Adloff et al. [H1 Collaboration], Eur. Phys. J. C 21 (2001) 33 arXiv:hep-ex/0012053.

[3] S. Chekanov et al. [ZEUS Collaboration], Eur. Phys. J. C 21 (2001) 443 arXiv:hep-ex/0105090.

[4] C. Adloff et al. [H1 Collaboration], Eur. Phys. J. C 30 (2003) 1 arXiv:hep-ex/0304003.

[5] S. Chekanov et al. [ZEUS Collaboration], Phys. Rev. D 70 (2004) 052001 arXiv:hep-ex/0401003.

[6] K. Golec-Biernat and M. Wüsthoff, Phys. Rev. D 59 (1998) 014017 arXiv:hep-ph/9807513.

[7] K. Golec-Biernat and M. Wüsthoff, Phys. Rev. D 60 (1999) 114023 arXiv:hep-ph/9903358.

[8] J. Bartels, K. Golec-Biernat and H. Kowalski, Phys. Rev. D 66 (2002) 014001 arXiv:hep-ph/0203258.

[9] M. McDermott, L. Frankfurt, V. Guzey and M. Strikman, Eur. Phys. J. C $\mathbf{1 6}$ (2000) 641 arXiv:hep-ph/9912547).

[10] H. G. Dosch, T. Gousset and H. J. Pirner, Phys. Rev. D 57 (1998) 1666 arXiv:hep-ph/9707264.

[11] A. I. Shoshi, F. D. Steffen and H. J. Pirner, Nucl. Phys. A 709 (2002) 131 arXiv:hep-ph/0202012.

[12] A. Donnachie and H. G. Dosch, Phys. Rev. D 65 (2001)
014019 arXiv:hep-ph/0106169.

[13] E. Iancu, K. Itakura and S. Munier, Phys. Lett. B 590 (2004) 199 arXiv:hep-ph/0310338.

[14] J. R. Forshaw and G. Shaw, JHEP 0412 (2004) 052 arXiv:hep-ph/0411337.

[15] J. R. Forshaw, R. Sandapen and G. Shaw, JHEP 0611 (2006) 025 arXiv:hep-ph/0608161.

[16] N. N. Nikolaev and B. G. Zakharov, Z. Phys. C 49 (1991) 607.

[17] N. N. Nikolaev and B. G. Zakharov, Z. Phys. C 53 (1992) 331.

[18] A. H. Mueller, Nucl. Phys. B 415 (1994) 373.

[19] V. N. Gribov, Sov. Phys. JETP 30 (1970) 709 [Zh. Eksp. Teor. Fiz. 57 (1969) 1306].

[20] B. L. Ioffe, Phys. Lett. B 30 (1969) 123.

[21] A. Donnachie, H. G. Dosch, P. V. Landshoff and O. Nachtmann, Pomeron Physics And QCD, Cambridge University Press, 2002.

[22] C. Ewerz and O. Nachtmann, Annals Phys. 322 (2007) 1635 arXiv:hep-ph/0404254.

[23] C. Ewerz and O. Nachtmann, Annals Phys. 322 (2007) 1670 arXiv:hep-ph/0604087.

[24] C. Ewerz and O. Nachtmann, Phys. Lett. B 648 (2007) 279 arXiv:hep-ph/0611076. 
[25] O. Nachtmann, Elementary Particle Physics: Concepts and Phenomena, Springer Verlag, Berlin, Heidelberg, 1990.

[26] L. N. Hand, Phys. Rev. 129 (1963) 1834.

[27] H. Abramowicz, E. M. Levin, A. Levy and U. Maor, Phys. Lett. B 269 (1991) 465.

[28] H. Abramowicz and A. Levy, arXiv:hep-ph/9712415.

[29] D. Schildknecht, in Proc. of the 15th International Workshop on Deep-Inelastic Scattering and Related Subjects (DIS 2007), eds. G. Grindhammer and K. Sachs, Munich, Germany (2007) arXiv:0707.3379.

[30] Encyclopedic Dictionary of Mathematics, eds. S. Iyanaga and Y. Kawada, vol. I, pp. 304 ff., MIT Press, Cambridge, Massachusetts, and London, England, 1980.

[31] B. Schölkopf and A. Smola, "Learning with Kernels", pp. 150 ff., MIT Press, Cambridge, MA, 2002.
[32] N. I. Ahiezer and M. Krein, "Some questions in the theory of moments", pp. 128 ff., American Mathematical Society, Providence, Rhode Island, 1962.

[33] W. I. Smirnov, "Lehrgang der höheren Mathematik", vol. V, pp. 15 ff. VEB Deutscher Verlag der Wissenschaften, Berlin, 1988.

[34] Mathematics Dictionary, eds. G. James and R. C. James, D. Van Nostrand Comp., Inc., Toronto, New York, London, 1949.

[35] We recall that for a given subset $S$ of $\mathbb{R}$ the infimum of the set $S$ is the greatest number less than or equal to each element of $S$. Similarly, the supremum is the smallest number that is greater than or equal to each element of $S$. For a compact set $S$ the minimum (maximum) coincides with the infimum (supremum). 